\title{
Enhanced adaptive code modulation for rainfall fade mitigation in Ethiopia
}

\author{
Eyob Mersha Woldamanuel ${ }^{1,2^{*}}$ and Feyisa Debo Diba ${ }^{1,2}$
}

*Correspondence:
eyob.mersha12@gmail.com
' School of Electrical
and Computer Engineering,
Haramaya Institute
of Technology (HiT),
Haramaya University,
138 Haramaya, Oromiya,
Ethiopia
Full list of author information
is available at the end of the
article

${ }^{*}$ Correspondence:

eyob.mersha12@gmail.com

${ }^{1}$ School of Electrical

and Computer Engineering,

Haramaya Institute

Haramaya University,

138 Haramaya, Oromiya

article

\begin{abstract}
Rain attenuation is considerably noticed in a frequency spectrum above 7-GHz for tropical equatorial regions and in a frequency spectrum higher than $10-\mathrm{GHz}$ for temperate climates. The attenuation prediction method provided by the International Telecommunication Union-Recommendation (ITU-R), through Recommendation P.530-16 and P.618-13 utilize data collected from temperate regions. Since the average raindrop size is bigger and the rainfall rate is high in magnitude in tropical regions than that of non-tropical areas, this prediction model is not suitable for the measured rain data. Unfortunately, a rain fade mitigation technique based on local rain data has not been adequately studied in tropical regions. This paper presents an enhanced adaptive code modulation (ACM) for rainfall fade mitigation in Ethiopia. In this research work, locally collected one-minute rain rate data is used to determine the rain attenuation. Then based on this result, the neuro-fuzzy inference system is employed to enhance the mitigation technique. Furthermore, a comparison of the performance of this proposed scheme is with the non-adaptive technique, and fuzzy-based adaptive modulation and coding technique is carried out. MATLAB simulation result showed that lower-order quadrature amplitude modulation (QAM) scheme with a lower convolutional coding rate is better in maintaining link availability in bad weather conditions. However, spectral efficiency is improved by utilizing a larger constellation size of quadrature amplitude modulation (QAM) scheme with a higher convolutional coding rate when the channel is not affected by rain.
\end{abstract}

Keywords: Adaptive code modulation (ACM), ITU-R, Neuro-fuzzy inference system, QAM, Rain attenuation, Rain rate

\section{Introduction}

Nowadays, the massive demand for efficient and reliable wireless communication systems has been obtained critical attention from researchers and network designers to study communication systems that operate at microwave and millimetric wavebands. The reason for this is the inability of the lower frequency spectrum to handle this increasing demand for large bandwidth and high channel capacity due to congestion $[1,2]$. However, the reliability of radio links at a higher operating frequency band can be affected by numerous atmospheric elements $[1,3]$. Of all atmospheric constituents, rainfall is the dominant cause of impairment at microwave and millimetric wavebands, party material in this article are included in the article's Creative Commons licence, unless indicated otherwise in a credit line to the material. If material is not included in the article's Creative Commons licence and your intended use is not permitted by statutory regulation or exceeds the permitted use, you will need to obtain permission directly from the copyright holder. To view a copy of this licence, visit http:// creativecommons.org/licenses/by/4.0/. 
which results in scattering, attenuation, and depolarization of signals at the receiver [4, 5].

Generally, attenuation due to rainfall, atmospheric gaseous, and frozen particles such as snow and ice crystals is very small and can be neglected in radio system design at frequencies of operation below 5-GHz. Though ITU-R recommends to consider rain attenuation in radio link design at a frequency of operation above 5- $\mathrm{GHz}$ [6], rain fades is quite noticed in tropical equatorial and temperate climates for a frequency spectrum higher than 7 and $10-\mathrm{GHz}$, respectively $[1,3]$. The experience of rain fade in relatively lower operating frequency in the former region than the latter one is due to the bigger raindrop size and higher rain intensity $[7,8]$. Recent researches also depict that for radio links operating at a frequency higher than $10-\mathrm{GHz}$ rainfall is the dominant factor that results in deep fading and network outage [9-13]. Droplets of rain that are found anywhere along the transmission path in between the radio links absorb and diffuse radio frequency. This absorption and diffusion of radio waves cause attenuation in the transmitted signal and reduction of the link availability.

Network performance analysis of wireless communications at higher radio frequencies depend on the assessment of rain attenuation to a great extent. The fade margin, that is, the system gain ensuring the necessary Quality of Service (QoS) against various transmission and other impairments must be significantly increased to compensate for the severe signal fading occurring at frequencies above 10-GHz. However, the larger fade margins required for fade compensation are not feasible either technically or economically. Under these circumstances, it is more difficult for microwave and millimeter-wave band communication systems to acquire the QoS specifications recommended by the Radio Communications Sector of the International Telecommunications Union (ITU-R) [14]. In order to satisfy the QoS specifications and to achieve high levels of link availability, rain fade countermeasures are required. The technique used to overcome this problem is known as Fade Mitigation Technique (FMT) $[15,16]$. Hence, the use of fade mitigation techniques to allow operations under lower fade margins is quite necessary.

The ITU-R provides basic Line-of-Sight (LOS) link design assumptions based on propagation prediction methods through Recommendation P.530-16 [6] and P.61813 [17]. This prediction method is prepared by utilizing data collected from temperate regions [18]. Since the average radius of raindrop and rainfall rate in tropical regions is greater than that of the non-tropical region, this prediction model is not suitable for tropical regions [5]. Owing to this, ITU-R recommends using locally measured rain data. Unfortunately, a rain fade mitigation technique based on local rain data has not been adequately studied in equatorial and tropical countries. This situation is more dominant in African equatorial and tropical countries. It is also evident that from research work carried out by Diba et al. [4], the ITU-R classification is not consistent with the rain rate values at different percentages of time for various locations of Ethiopia. It is therefore essential that for these particular regions, experimentally obtained parameters must be employed to modify or refine these propagation prediction methods. Thus, a rain attenuation countermeasure based on the local rain data model must be investigated for frequency spectrum above 10-GHz radio links. This is especially imperative for African tropical countries like Ethiopia where there are a high rainfall rate and intensity. Investigation of the mitigation technique of rain fade on microwave and millimeter-wave LOS 
terrestrial communication in Ethiopia based on local data is the main motivation for this proposed work.

Since the condition of the wireless channel is varying with time, an intelligent adaptive technique, which is good in decision-making, is required. In other words, due to the complexity, uncertainty, and adaptive nature of the wireless channel, the conventional non-intelligent systems cannot cope with an adaptive environment. Soft computing techniques such as fuzzy logic, neural networks, and neuro-fuzzy systems are preferred over the non-adaptive and fixed coding and modulation techniques in decision-making. Even though, several works have been done to investigate mitigation of rain fades in [19-22], most of the studies were focused on satellite links and not based on the local rain data. Beside this, one can encounter several studies in the area of adapting orthogonal frequency division multiplexing (OFDM) wireless links using fuzzy and neuro-fuzzy techniques [23-25]. However, the adapting capability of soft computing-based ACM technique to mitigate the effects of rain fade in single-frequency carrier communication link has not been adequately studied. This is also another motive of this study.

\section{Methodology}

In this section, a method used to calculate rain attenuation and neuro-fuzzy-based ACM is presented. A rainfall attenuation based on locally recorded rain data is determined and used as input to mitigate the rain fade. An adaptive coding and modulation schemebased controller using the neuro-fuzzy system that attains the desired bit error rate (BER) performance and channel data rate is investigated.

In order to mitigate fading over a time-varying channel, the neuro-fuzzy controller is first employed to select the desired modulation-coding rate pair. This selection is done to attain the highest data rate at the receiver end while achieving the target BER. The transmitter then adapts its coding rate and constellation size depending on the channel parameters to improve the performance of wireless systems.

\subsection{Rainfall attenuation determination}

According to ITU-R rain attenuation prediction procedures [6], the efficacious way of determining cumulative rainfall distribution is done by making a direct measurement at the site of interest. Thus, a two-year (October 2016-September 2018) one-minute rainfall rate recorded with a device installed at Jimma University, Ethiopia is utilized in this research work. The device that used to record the rain data was David Vantage Vue weather station. This equipment consists of an outdoor unit, a wireless Integrated Sensor Suite (ISS), that collects rainfall data at one minute integration time (every $60 \mathrm{~s}$ ), using collector-type tipping spoon which has a resolution of $0.2 \mathrm{~mm}$ per tip. The device record all the day with one-minute integration time. Both rainy and clear sky (zero rain) time rain intensity were measured. The rain-intensity values which is different from zero were assorted separately and analyzed. Following this, mathematical formulation pertaining to rain-related calculation was employed to compute the required parameters.

Depending on the climatic and geographical conditions several rain attenuation models are available. The major ones are Crane global model, Two-component model, Simple Attenuation model, Garcia model, ITU-R model, Bryant model, and Moupfouma model. In this research work, the ITU-R rain attenuation model is used to determine rain fade. 


\section{ITU-R P.530-17 Model}

The ITU-R P.530-17 [6] gives a simple procedure that may be used for predicting the long-term data of rain attenuation. The following simple procedure is presented in this model for approximating the rain attenuation data which is recorded in the long run:

Step 1: Rain rate $R_{0.01}$ exceeded for $0.01 \%$ of the time (with an integration time of 1 $\min$ ) is calculated.

Step 2: Specific rain attenuation $\left(\gamma_{R}\right)$ specified in $(1),(\mathrm{dB} / \mathrm{km})$ is computed for desired frequency, polarization and rain rate based on Recommendation ITU-R P.838-3 [26].

$$
\gamma_{R}=K R^{\alpha}
$$

Step 3: Calculate the effective path distance, $d_{\text {eff }}$, of the link by multiplying the actual path distance $d$ by a link path reduction factor $r$. The value of $r$ is approximated by:

$$
r=\frac{1}{0.477 d^{0.633} R_{0.01}^{0.073 \alpha} f^{0.123}-10.579\left(1-e^{-0.024 d}\right)}
$$

where $\mathrm{f}(\mathrm{GHz})$ is the frequency and $\alpha$ is the constant coefficient of vertical polarization from Step 2. It is recommended that the highest value of $\mathrm{r}$ is 2.5. Subsequently, in case the denominator of (2) is below 0.4 , it is advised to use this highest value for $\mathrm{r}$.

Step 4: The approximate total link attenuation due to rain surpassed for $0.01 \%$ of the time is computed as:

$$
A_{0.01}=\gamma_{R} d_{e f f}=\gamma_{R} \times d \times r
$$

Step 5: The attenuation exceeded for other percentages of time $\mathrm{p}$ in the range $0.001 \%$ to $1 \%$ may be deduced from the following power law:

$$
\begin{aligned}
& \frac{A_{p}}{A_{0.01}}=C_{1} P^{-\left(C_{2}+C_{3} \log _{10} P\right)} \\
& C_{1}=\left(0.007^{C_{0}}\right)\left[0.12^{1-C_{0}}\right] \\
& C_{2}=0.855 C_{0}+0.546\left(1-C_{0}\right) \\
& C_{3}=0.139 C_{0}+0.043\left(1-C_{0}\right) \\
& C_{0}= \begin{cases}0.12+0.4\left[\log _{10}(f / 10)^{0.8}\right], & f \geq 10 \mathrm{GHz} \\
0.12, & f<10 \mathrm{GHz}\end{cases}
\end{aligned}
$$

Step 6: Worst-month data are determined by computing the annual time percentages $\mathrm{p}$ corresponding to the worst-month time percentages $p_{w}$ using climate information stated in ITU-R P.841-5 [27]. The magnitude of A surpassed for percentages of the time $\mathrm{p}$ on an annual basis will be exceeded for the corresponding percentages of time $p_{w}$ on a worstmonth basis.

Values of coefficient $\mathrm{k}$ and $\alpha$ in (2) are frequency, $\mathrm{f}(\mathrm{GHz})$, dependent in the spectrum 1 to 1000-GHz. Table 1 depicts a selected frequency of operation [26] with their associated coefficients $\mathrm{k}$ and $\alpha$ for vertical polarization. These frequencies are selected from rain-induced 
Table 1 Coefficients for $K_{v}$ and $\alpha_{v}$ for indicated frequency of operation

\begin{tabular}{lll}
\hline Frequency $(\mathrm{GHz})$ & $K_{V}$ & $\alpha_{V}$ \\
\hline 11 & 0.01731 & 1.1617 \\
15 & 0.05008 & 1.0440 \\
28 & 0.1964 & 0.9277 \\
38 & 0.3844 & 0.8552 \\
45 & 0.5375 & 0.8123 \\
60 & 0.8515 & 0.7486 \\
72 & 1.0561 & 0.7171 \\
\hline
\end{tabular}

attenuation affected microwave frequency range (frequency higher than $10-\mathrm{GHz}$ ) and millimeter-wave (mmWave) bands, which is the spectrum roughly from 30 to $300-\mathrm{GHz}$. Of the selected frequencies 28,38 , and $60-\mathrm{GHz}$, are considered as leading candidates for $5 \mathrm{G}$ system [28].

\subsection{Radio link performance analysis}

Terrestrial LOS radio link performance analysis depends on the calculations of the link budget. The link budget computation involves the calculation of received signal level, fade margin, and noise figure [29]. The expected received signal power $P_{r}$ is computed using the following formula.

$$
P_{r}=P_{t}+G_{t x}+G_{r x}-F S L-L \quad(\mathrm{~dB})
$$

where $P_{t}$ is the transmitter power in output at the antenna input. This is the amount of microwave carrier output power, usually expressed in $\mathrm{dBm}$; $\mathrm{L}$ is losses due to the presence of atmospheric gases, vegetation, buildings, clouds, and fogs; $G_{t x}=$ Transmit antenna gain, $G_{r x}=$ Receive antenna gain, FSL $=$ Free space path loss.

The performance of a communication system is estimated based on the achievable signal-to-noise (SNR) at the receiver. The term SNR (in $\mathrm{dB}$ ) refers to the estimation of signal strength as a function of signal degradation and background noise. This power can be expressed as [13]

$$
\frac{S}{N}=\frac{C}{N}=\frac{\left(P_{t} G_{t x} G_{r x}\right)}{\left(K T B L_{s y s}\right)}\left(\frac{4 \pi l}{\lambda_{0}}\right)^{2}
$$

where $L_{s y s}$ is system loss at the receiver and transmitter; $\lambda_{0}=$ free-space wavelength $(\mathrm{m})$ which is determined from frequency as $\left(\lambda_{0}=\frac{0.3}{f(\mathrm{GHz})}\right)$; Gtx and Grx are transmitting and receiving antenna gain respectively; $K$ is the Boltzmann's constant $=1.38 \times 10^{-23} \mathrm{~J} / \mathrm{K} ; B$ $=$ bandwidth $(\mathrm{Hz})$; and $l$ is the link distance $(\mathrm{m}) . T$ is the noise temperature $(\mathrm{K})$ of the system which is assumed to be $290 \mathrm{~K}$.

Receiver antenna noise figure, NF, value is computed as:

$$
\begin{aligned}
& N F=K T B(w) \\
& N F=174(\mathrm{dBm})-10 \log _{10} B(\mathrm{~Hz})
\end{aligned}
$$


Equation (10) can equivalently be computed in unit decibel (dBm) as [13]:

$$
\begin{aligned}
\frac{S}{N}= & P_{t}+G_{t x}+G_{r x}-F S L(\mathrm{~dB})-L(\mathrm{~dB}) \\
& -10 \log _{10} B(\mathrm{dBm} \mathrm{Hz})+174(\mathrm{dBm}) \\
\frac{S}{N}= & P_{r}-10 \log _{10} B(\mathrm{~Hz})+174(\mathrm{dBm})
\end{aligned}
$$

where $P_{r}$ is determined from the link profile given parameters.

In real-world radio links, for the communications system to acquire the least tolerable quality of service (usually termed as minimum SNR), the received power level often needs to be better than the threshold level. When the received signal magnitude is below the threshold value, network outage will occur. As a result, the radio link performance evaluation is done by comparing the percentage of network outage time with the total time duration. This design allowance for received power is termed as the link margin and defined as the difference between the design value of received power and the minimum threshold value of receive power [29]:

$$
\operatorname{LinkMargin}(\mathrm{dB})=L M=P_{r}-P_{r}(\min )
$$

where all quantities are in $\mathrm{dB}$. Link margin that is used to account for fading effects is sometimes referred to as fade margin.The fade margin is thus,

$$
\text { FadeMarigin }=R_{X T H}-P_{r}
$$

In this case FadeMargin $=$ LinkMargin, and $R_{X T H}=P_{r}(\min )$

\subsection{SNR calculation}

The SNR of the particular radio link whose radio link profile has been defined in Table 2 is computed as (10). The computation is based on the values given in the link profile and the result from rain attenuation determination using ITU-R model.

\subsubsection{Clear air SNR calculation}

The received signal for clear sky condition is determined using (9). Substituting the values from the link profile table, Table 2, the SNR of this link at a clear sky environment can be determined using (17) as :

$$
\frac{S}{N}=P_{r}-B(\mathrm{~dB} \mathrm{~Hz})+174(\mathrm{dBm})
$$

where $B$ is the bandwidth of the radio link which is given as $21.7 \mathrm{MHz}$ in Table 2 .

\subsubsection{Rainy time SNR calculation}

Here the received signal is additionally degraded by the total rain attenuation amount. Thus, SNR at rainy time is calculated as [13]

$$
\frac{S}{N}=\left(P_{r}-A_{\text {rain }}\right)-B(\mathrm{~dB} \mathrm{~Hz})+174(\mathrm{dBm})
$$


Table 2 Link parameters for terrestrial line of sight networks

\begin{tabular}{lll}
\hline Parameters & Link & Link \\
& Jimma & Muja \\
\hline Antenna height & 10 & 10 \\
Antenna gain (dBi) & 42 & 42 \\
Tx line unit loss (dB/100) & 4.53 & 4.53 \\
Tx line loss & 0.91 & 0.91 \\
Frequency (GHz) & 11 & 11 \\
Circuit branching loss (dB) & 6.8 & 6.8 \\
True azmuth & 203.92 & 23.91 \\
Vertical angle & 1.48 & -1.59 \\
Link distance $(k m)$ & 13.4 & \\
Free space loss (dB) & 139.6 & \\
Rx threshold level (dBm) & -76.2 & \\
Effective frequency spacing (MHz) & 21.7 & \\
Atmospheric absorption loss (dB) & 0.35 & \\
Tx power (dBm) & 30 & \\
\hline
\end{tabular}

where $A_{\text {rain }}$ is the attenuation induced by rain. If the SNR at rain rate of exceedance $0.01 \%$ is required, it can be calculated by replacing $A_{\text {rain }}$ by total rain attenuation at rain rate of exceedance $A_{0.01}$. Finally, the SNR for different fading depth (rain rate) is computed.

The link profile parameters presented in Table 2 was obtained from Ethiotelecom, the only telecoms service provider in Ethiopia. The microwave antenna installed in LoS in between Jimma and Mujja are the core microwave link of Ethiotelecom. Jimma is a town located at aerial distance of $261 \mathrm{~km}$ southwest of Addis Ababa, the capital city of Ethiopia. Mujja is a place $13.4 \mathrm{~km}$ away from the town of Jimma.

\section{Implementation of adaptive coding and modulation}

According to the proposed block diagram indicated in Fig. 1, the randomly generated data source is encoded by exploiting a feed-forward convolutional encoder with different coding rates. Then, this convolutionally encoded data is modulated by M-ary QAM. Following this, the encoded and modulated symbols are transmitted. Finally, white Gaussian noise is added to the transmitted signal and rain-induced attenuation is also taken into consideration.

At the receiver side, after conversion of the analog signal back to a digital format, the received data is demodulated using an adaptive MQAM demodulator. Following this, the convolutionally encoded bits are decoded using Viterbi decoder so as to remove the redundant bits added for the purpose of error correction.

The choice of modulation scheme mainly relies on the channel characteristics. If the amplitude of the received signal is varied rapidly, QAM and pulse amplitude modulation (PAM) may be particularly vulnerable because a wideband automatic gain control (AGC) must be employed to reimburse for the channel variations. In such a case, phase shift keying (PSK) or differential phase shift keying (DPSK) is more suitable, since the information is conveyed by the signal phase and not by the signal amplitude [30]. However, in MQAM the information bits are encoded in both the amplitude and phase of 


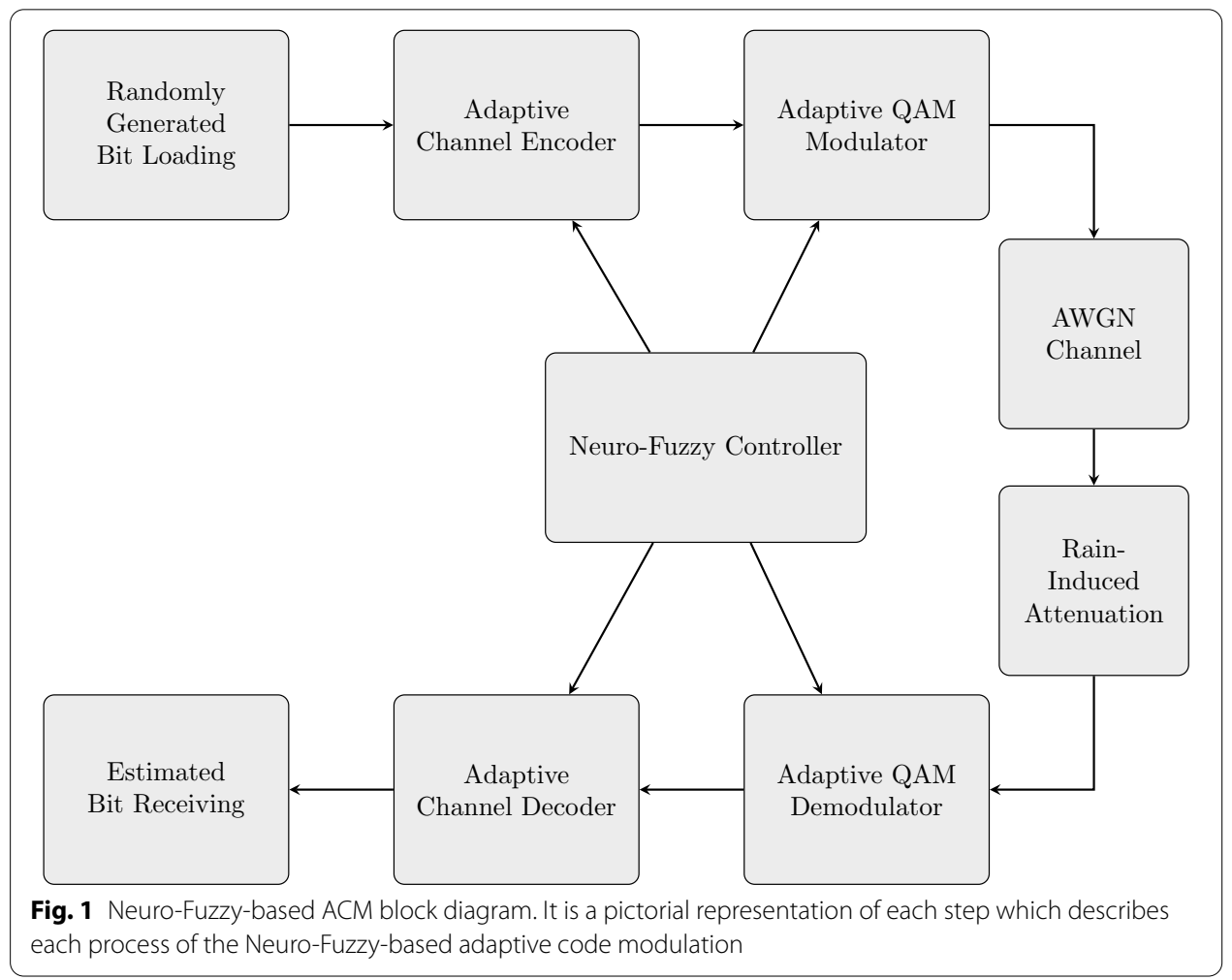

the transmitted signal. Owing to this, MQAM has two degrees of freedom compared to both M-ary PAM (MPAM) and M-ary PSK (MPSK) that have one degree of freedom to encode the information bits (amplitude or phase). As a result, MQAM is more spectrally efficient than MPAM and MPSK, in that it can encode the greatest number of bits per symbol for given average energy [31]. For this reason, MQAM is selected as a modulation scheme in this study.

Practically, the designed system is incapable of regenerating the transmitted message signal as it is because of the noise plus rain-induced attenuation superimposed on the wireless medium. It is obvious to have some bits received in error. By changing the modulation order and coding rate BER is computed for each SNR based on the system parameters shown in Table 4. Comparison of the performance of BER for ACM techniques is studied. A comparison of adaptive modulation with fixed coding and ACM is also analyzed.

\subsection{Neuro-fuzzy-based ACM design}

Reference [32] describe the neuro-fuzzy system as a combination of neural network and fuzzy inference system (FIS). It has the combined benefits of the two soft computing systems. It inherits the neural learning method utilized in adjusting the membership function parameters from the neural network and the structure from FIS. Usually, it has the benefit of permitting an easy transformation of the last system into a set of if-then rules. The fuzzy system can also be seen as a neural network structure with knowledge distributed throughout connection strengths [32, 33]. 
Using this hybrid soft computing method, an initial fuzzy logic model with its input parameters is first obtained from the input-output data of the BER performance simulation of different modulation-code pairs. A neural network is then applied to update the initialized fuzzy rules and membership functions to create the final neuro-fuzzy method. In this neuro-fuzzy approach, back-propagation learning and the least-squares method are employed to update membership functions and adjust design parameters respectively. The general neuro-fuzzy system approach flowchart is shown in Fig. 2.

In the following section, the steps which are incorporated in the proposed neuro-fuzzy system model will be discussed briefly. Generation of Input/Output (I/O) data pairs, spectral efficiency optimization, and neuro-fuzzy architecture for ACM is presented in this section. Finally, this section is completed by describing how the designed adaptive neuro-fuzzy inference system (ANFIS) is trained.

\subsubsection{Generation of input/output data pairs}

The proposed neuro-fuzzy system must be trained by a manually generated data from the graph of each BER performance simulation of different modulation-code pairs. Figure 3 shows how we get the desired coding rate, code-rate 1/3 for this particular example, and different modulation order-BER plot coordinate that fulfill different target bit error rate values such as $10^{-6}, 10^{-5}, 10^{-4}, 10^{-3}$, and $10^{-2}$.

These pairs are obtained using two methods: one is by drawing a straight line from the given SNR to the target quality of service points; the other one is by running a MATLAB command called "ginput" and take the value of the coordinate at a target BER value. The output is taken as the product of code-modulation pairs.

Table 3 shows a sample of I/O data pairs that are obtained as a function of SNR, BER, modulation order, and coding rate to select the best modulation and coding rate to maximize the spectral efficiency of the wireless system. All the input-output data pairs are not important; only those that maximize the throughput are taken based on the spectral efficiency optimization. The input-output relationship of the data in Table 3 is governed by (19).

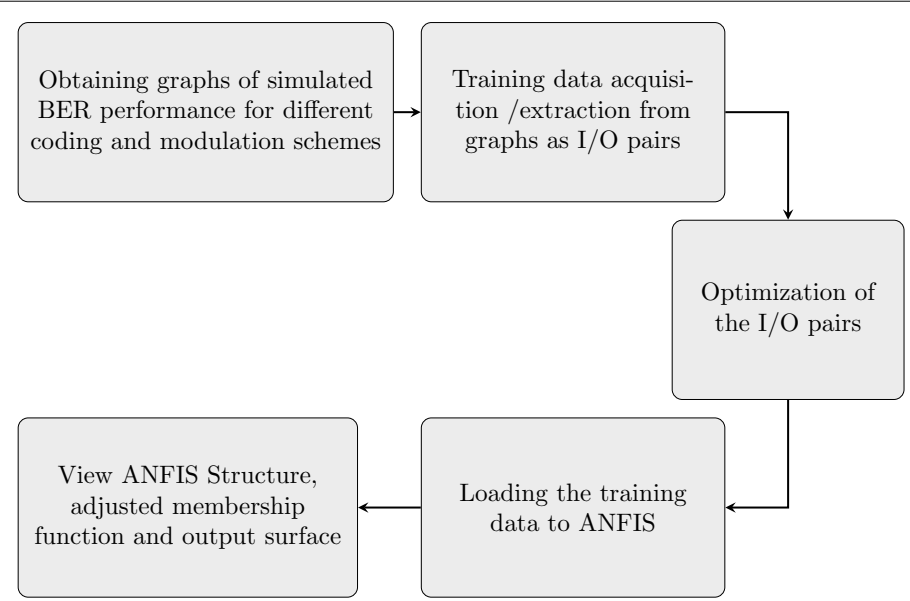

Fig. 2 Neuro-Fuzzy-based system model flow chart. A flow chart which describes neuro-fuzzy system which enhances the adaptability capacity of the adaptive code modulation system 


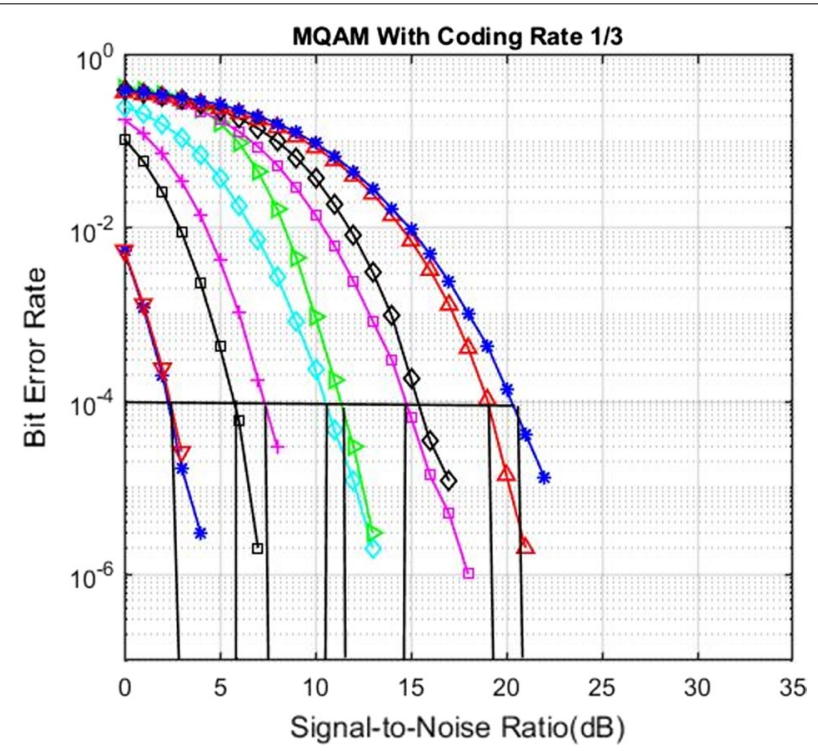

Fig. 3 Generation of I/O pairs for different modulation schemes with 1/3 code-rate. Shows how we get the desired coding rate, code-rate 1/3 for this particular example, and different modulation order-BER plot coordinate that fulfill different target bit error rate values such as $10^{-6}, 10^{-5}, 10^{-4}, 10^{-3}$, and $10^{-2}$

Table 3 Sample of I/O data pairs obtained from graph resulted from simulation

\begin{tabular}{lllll}
\hline Input & & & & Output \\
\hline SNR (Eb/No) (dB) & BER & Modulation type & Coding rate & $\begin{array}{l}\text { Max. data } \\
\text { rate (bits/s/ } \\
\text { Hz) }\end{array}$ \\
\hline 3.5 & & & $1 / 4$ & 0.5 \\
4.5 & $10^{-3}$ & 4 QAM & $1 / 3$ & 1 \\
9.9 & $10^{-3}$ & 8 QAM & $1 / 2$ & 2 \\
12.9 & $10^{-4}$ & 16 QAM & $2 / 3$ & 3.33 \\
16.5 & $10^{-2}$ & 32 QAM & $3 / 4$ & 4.5 \\
20.7 & $10^{-3}$ & 64 QAM & $2 / 3$ & 4.667 \\
24 & $10^{-2}$ & 128 QAM & $2 / 3$ & 5.33 \\
25.6 & $10^{-4}$ & 256 QAM & $3 / 4$ & 6.75 \\
27.45 & $10^{-4}$ & 512 QAM & $2 / 3$ & 5.33 \\
29.8 & $10^{-6}$ & 256 QAM & $3 / 4$ & 7.5 \\
31.5 & $10^{-5}$ & 1024 QAM & $2 / 3$ & 6.67 \\
32.96 & $10^{-5}$ & 1024 QAM & $2 / 3$ & 6 \\
\hline
\end{tabular}

\subsubsection{Spectral efficiency optimization}

Communication network designers often aims to have a radio link that operates at or below a specified BER with the highest achievable data rate. This enables the radio link to achieve the best achievable QoS. Assuming constant transmit power, optimization of spectral efficiency $(\eta)$ for ACM is given by [34]:

$$
\max \eta=R_{C} \log _{2}(M) \text { such that } \overline{B E R}(\bar{\Upsilon}) \leq B E R_{T}
$$


where $\bar{\Upsilon}$ is average SNR, $R_{C}$ is code rate, $\overline{B E R}$ is average $B E R$ and $M$ is the modulation order.

In order to achieve the maximum throughput in the ACM scheme, the following consideration should take into account:

iFor the same BER and SNR pair, better throughput is selected.

ii For the same data rate, less modulation and coding rate is chosen that demand less SNR.

iiiThe lookup table scheme may not have a complete number of data pairs, then those missed parts are completed by ANFIS.

\subsubsection{ANFIS architecture for ACM}

In this paper, a type of neuro-fuzzy method termed as Adaptive Network-based Fuzzy Inference System (ANFIS) is exploited as a modeling approach. MATLAB neuro-fuzzy designer app has been used to carry out and examine the ANFIS system as a development tool. The tool consists of a fuzzy logic designer, membership function editor, rule editor, neuro-fuzzy designer rule, and surface viewers.

The fuzzy logic designer is a GUI tool that illustrates general information of a FIS. The membership function editor displays and edits membership functions of all the inputoutput variables. The rule editor allows a designer to build fuzzy rules automatically. The rule viewer gives better description and interpretation of all the FIS rules.

The neuro-fuzzy designer has several features. It is utilized to load FIS training data, save the trained FIS, open a new Sugeno type system, generate the FIS, and view the ANFIS structure. This app has graphical user interfaces (GUIs) to interpret the trained FIS model and test and validate the ANFIS model using other data. It can also allow loading the test data for validation purposes, load the predefined FIS designed by the Fuzzy Logic designer, plot the loaded train data, plot the train data against the test data for comparison. The output surface viewer is a three-dimensional plot that represents a mapping of input variables to the output variable.

\subsubsection{ANFIS system for training process}

Enhancement of data rate using ANFIS has been developed and examined as depicted in Fig. 2. The architecture of the ANFIS used to achieve the spectral efficiency has been developed and investigated as shown in Fig. 4. It consists of five layers corresponding to various functions. The proposed model is trained with SNR, BER, coding rate and modulation order as inputs and data rate as an output which is generated from simulations using parameters depicted in Table 4.

Both the fuzzy logic system principles and learning capabilities of neural networks are being employed to construct ANFIS. At the initial stage, a basic fuzzy logic system controller is built to utilize the linguistic fuzzy rules. Then, the I/O data pairs are used to train the ANFIS controller. 
Table 4 System parameters and values

\begin{tabular}{ll}
\hline Schemes & Parameter values \\
\hline Modulation order & 4 QAM, 8 QAM, 16 QAM, 32 QAM, 64 QAM, \\
SNR & 128 QAM, 256 QAM, 512 QAM and 1024 QAM \\
Target BER & 0 to 33 dB \\
Coding-rate & $10^{-6}$ to $10^{-2}$ \\
Spectral efficiency & $1 / 4,1 / 3,1 / 2,2 / 3$, and 3/4 \\
Constraint length & 0.5 to $7.5 \mathrm{bits} / \mathrm{s} / \mathrm{Hz}$ \\
Channel model & 3 \\
\hline
\end{tabular}

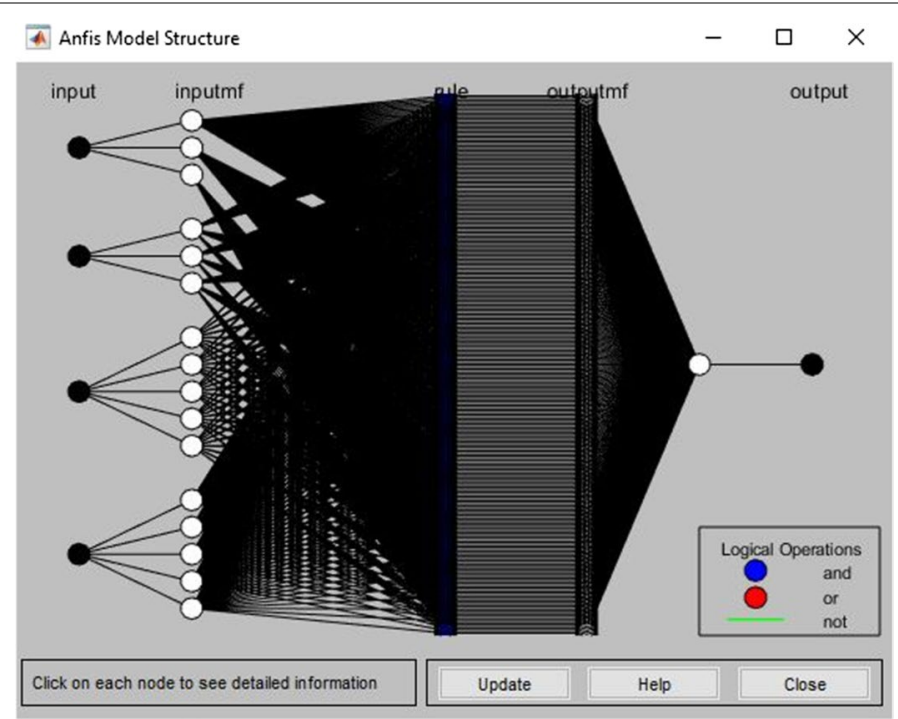

Fig. 4 ANFIS structure with four inputs and one output. A diagram constructed in MATLAB neuro-fuzzy designer apps which depicts ANFIS architecture with four inputs and one output

The steps involved in the ANFIS training process are:

iLoading the I/O training data;

ii Generate an initial fuzzy inference system model;

iii View the FIS model structure;

iv Select the FIS model optimization method (hybrid method);

$\mathrm{v}$ Choose the training epochs and training error tolerances;

viTrain ANFIS and view adjusted membership functions and the output surface.

Finally, after the FIS training process is completed validation of the trained system must be carried out. The trained FIS is validated using testing data that differs from the one we used to train the FIS. In this research work, validation is done using the data that is generated using the MATLAB data extraction method. These test data is a BER value for each integer value of SNR in the range 0 to $33 \mathrm{~dB}$ and the associated modulation code pair and data rate. 
The range of fuzzy variables for the BER input values given by $10^{-6}, 10^{-5}, 10^{-4}, 10^{-3}$, and $10^{-2}$ should be spaced equally and quantifiable. To get this a logarithmic operation is performed as given in the following equation [34]

$$
\begin{aligned}
& B E R=\log _{10} 10^{-p}, \quad p=2,3,4,5,6 \\
& B E R=-p
\end{aligned}
$$

In this paper, 225 first-order Sugeno-type fuzzy inference rules have been constructed as in the form presented in Eqs. (21)-(23) for the proposed neuro-fuzzybased ACM. In other word, the multiplication of the number of membership function of the four input parameters( SNR, BER, coding rate, and modulation order) result in 225 fuzzy linguistic rules. i.e $3 \times 3 \times 5 \times 5=225$ rules. The general rule:

$$
\begin{aligned}
& \text { if } x_{1} \text { is } A_{i 1} A N D x_{2} \text { is } A_{i 2} \text { AND } \\
& x_{3} \text { is } A_{i 3} A N D x_{4} \text { is } A_{i 4} \text { THEN } \\
& f_{1}=p_{i} x_{1}+q_{i} X_{2}+t_{i} x_{3}+s_{i} x_{4}+r_{1} i
\end{aligned}
$$

The specific rules:

$$
\begin{aligned}
& \text { IF } x_{1} \text { is } A_{11} \text { AND } x_{2} \text { is } A_{12} \text { AND } \\
& x_{3} \text { is } A_{13} A N D x_{4} \text { is } A_{14} \text { THEN } \\
& f 1=p_{1} x_{1}+q_{1} x_{2}+t_{1} x_{3}+s_{1} x_{4}+r_{1} \\
& \text { IF } x_{1} \text { is } A_{21} \text { AND } x_{2} \text { is } A_{22} \\
& \text { AND } x_{3} \text { is } A_{23} \text { AND } x_{4} \text { is } A_{24} \text { THEN } \\
& f 1=p_{2} x_{1}+q_{2} x_{2}+t_{2} x_{3}+s_{2} x_{4}+r_{1}
\end{aligned}
$$

where:

i $p_{i}, q_{i}, t_{i}, s_{i}$, and $r_{i}$ are design parameters,

ii $f_{i}$ are the outputs within the fuzzy area specified by the fuzzy logic rules,

iii $A_{i j}$ are the fuzzy sets/membership functions for each input variables, and iv $x_{i}$ is the input parameters to the neuro-fuzzy system and $\mathrm{i}=1,2,3, \ldots$

Layer 1-Input node: Each node in this layer is an input node, that corresponds to one input parameter. These nodes pass the input signals to the layer 2 . The proposed fuzzy sets have three membership function for the input variables SNR and BER designated as low, medium and high and five membership function for the input variable modulation order (MOD) and code rate represented as very low, low, medium, high, and very high. The output of the neuron $\mathrm{i}$ in the input node is obtained as:

$$
O_{i}^{1}=f_{i}^{1}\left(\text { net }_{i}^{1}\right)=\text { net }_{i}^{1}
$$

where $n e t_{i}^{1}$ is the $i$ th input to the node of layer one.

Layer 2-Input membership layer: Each node in this layer acts as a linguistic label of one of the input variables in input node, i.e., specifies the membership functions 
for each input parameters. The generalized bell-shaped membership function is used to represent each fuzzy set variables. The output of neuron $\mathrm{j}$ in layer 2 is given by:

$$
O_{j}^{2}=f_{j}^{2}\left(n e t_{2}^{2}\right)=\frac{1}{1+\left(\frac{x-C_{j}}{a_{j}}\right)^{2 b_{j}}}
$$

where $a_{j}, b_{j}$ and $c_{j}$ are parameters set that define shapes of $j$ th membership function. The parameter $b_{j}$ is usually positive and the parameter $c_{j}$ locates the center of the curve.

Layer 3-Rule layer: All the nodes in this layer calculates the firing strength of a rule via multiplication. Each node takes four inputs, to form 225 nodes in layer 3 and creates a fuzzy rule for all input variables. The output of the neuron $\mathrm{k}$ is obtained as follows:

$$
\begin{aligned}
& O_{k}^{3}=f_{k}^{3}\left(n e t_{k}^{3}\right)=n e t_{k}^{3} \\
& n e t_{k}^{3}=\prod_{j} w_{j k}^{3} y_{j}^{3}
\end{aligned}
$$

where $y_{j}^{3}$ is $j$ th input to the node layer 3 and $w_{j} k^{3}$ is assumed to be unity.

Layer 4-Output membership function: Neurons in this layer represent fuzzy sets used in the consequent fuzzy inference rules. An output membership neuron receives inputs from the corresponding fuzzy rule neuron and combines them by using the fuzzy operation union. The output of neuron $m$ is given by:

$$
\begin{aligned}
O_{m}^{4} & =f_{m}^{4}\left(n e t_{k m}^{4}\right)=\max \left(n e t_{k m}^{4}\right) \\
n e t_{k m}^{4} & =O_{k}^{3} w_{k m}
\end{aligned}
$$

where $w_{k m}$ is the output action of the $m$ th output associated with $k$ th rule.

Layer 5-Defuzzification layer: In this layer the sum-product composition is used to find the defuzzified output, i.e., crisp value. It calculates the output as the weighted average of the centroids of all output membership functions.

$$
\begin{aligned}
O_{o} & =f_{o}^{5}\left(n e t_{o}^{5}\right)=n e t_{o}^{5} \\
n e t_{o}^{5} & =\frac{\sum_{m} O_{m}^{4} a_{c m} b_{c m}}{\sum_{m} O_{m}^{4} b_{c m}}
\end{aligned}
$$

where $a_{c m}$ and $b_{c m}$ are centers and widths of the output fuzzy sets respectively. The value of $b_{c m}$ is assumed unity.

The Sugeno type FIS editor with four inputs and one output is shown in Fig. 5.

All input variables are specified by membership functions. A membership function is a curve that maps each input element to a membership value between 0 and 1 . In the designed ANFIS system, because of its smoothness, bell-shaped membership functions are considered for all $\mathrm{I} / \mathrm{O}$ variables.

The number of membership functions is chosen so as to cover the entire input space. For SNR input, low, medium, and high membership functions are considered.

The input parameter BER has also three membership functions designated as low, medium, and high which cover equally spaced value in between -6 to -2 as indicated in (20). For the modulation order input, five membership functions are taken namely 


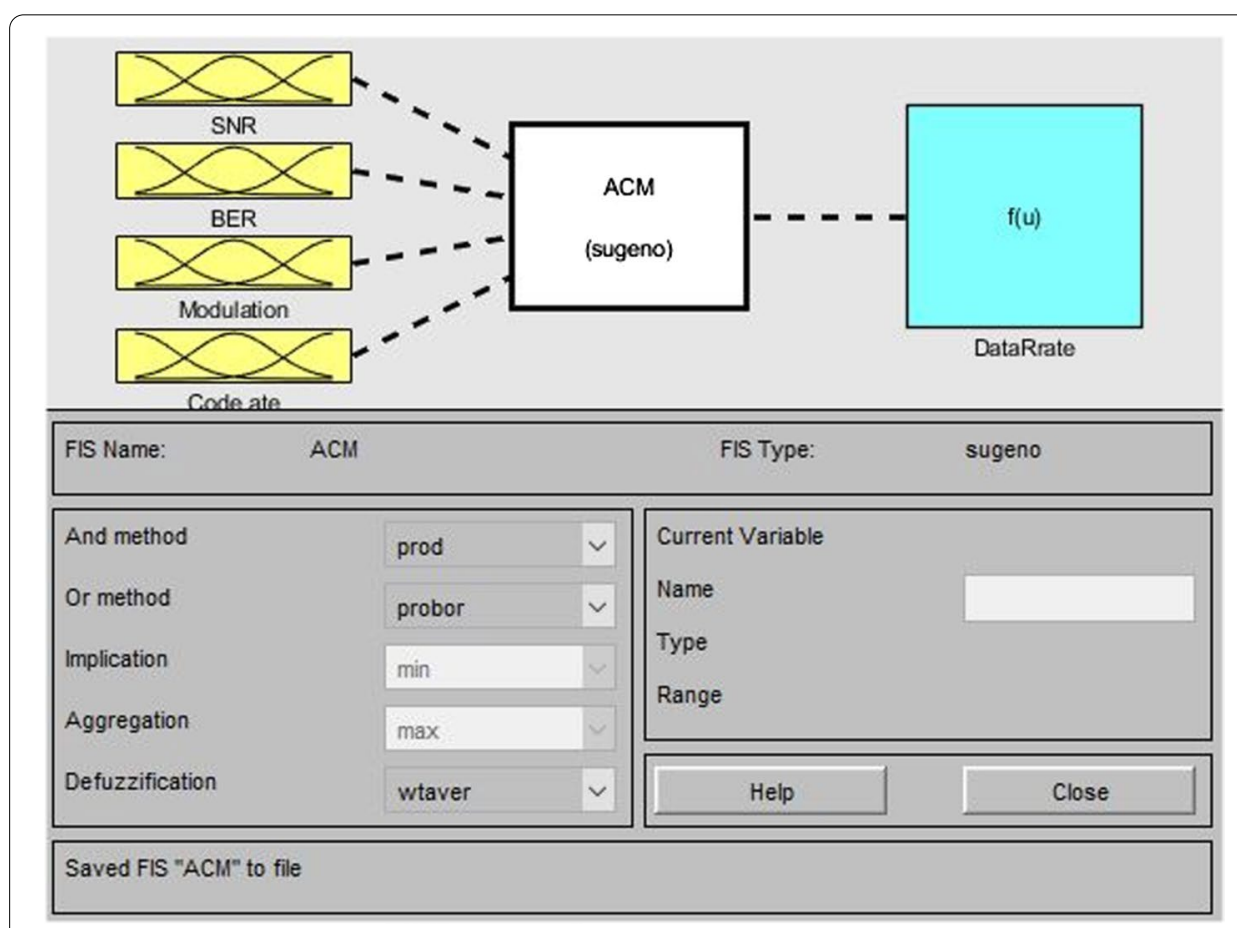

Fig. 5 Sugeno type FIS with 4 inputs and one output. A graphical user interface diagram which shows Sugeno type fuzzy inference system with four inputs and one output

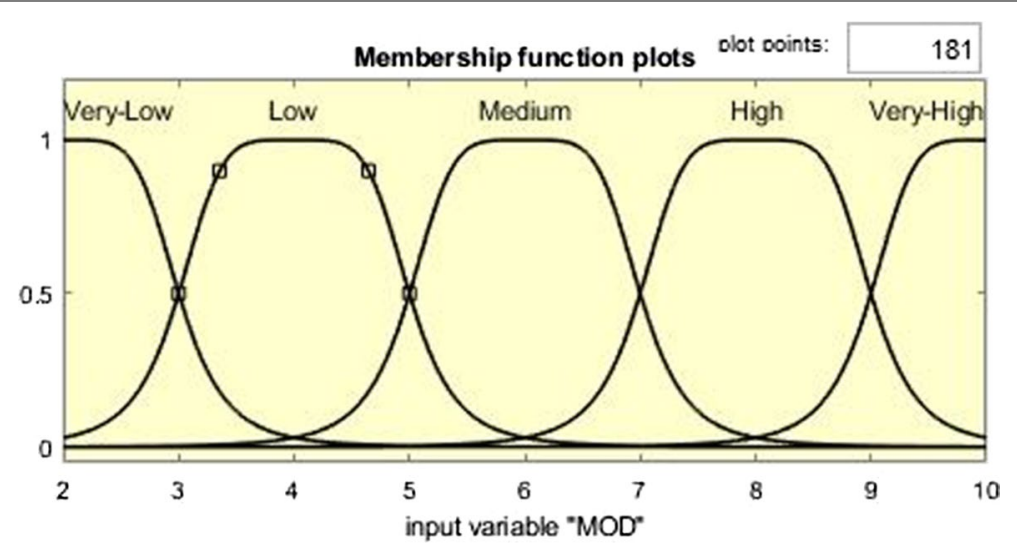

Fig. 6 Membership functions of input modulation. A graphical user interface diagram which shows membership functions of input modulation order

very low, low, medium, high, and very high. The modulation schemes are 4, 8, 16, $32,64,128,256,512$, and 1024 QAM with 1, 2, 3 to 9 number of bits per each QAM modulation scheme, respectively. As an example Fig. 6 illustrates the membership function of modulation order input.

The input variable code rate have a range extending from 0.25 to 0.75 . It contains five membership functions like input variable modulation type designated as 
Table 5 Specific and total rain attenuation values at $R_{0.01}$ and path distance $13.4 \mathrm{~km}$

\begin{tabular}{lcc}
\hline Frequency $(\mathrm{GHz})$ & Specific rain attenuation $\left(\gamma_{R}\right)(\mathbf{d B} / \mathbf{K m})$ & $\begin{array}{l}\text { Total rain } \\
\text { attenuation } \\
\left(A_{0.01}\right)(\mathbf{d B})\end{array}$ \\
\hline 11 & 4.201 & 27.65 \\
15 & 6.97 & 46.14 \\
28 & 15.768 & 95.71 \\
38 & 21.91 & 129.19 \\
45 & 25.01 & 145.49 \\
60 & 29.32 & 165.50 \\
72 & 31.33 & 172.40 \\
\hline
\end{tabular}

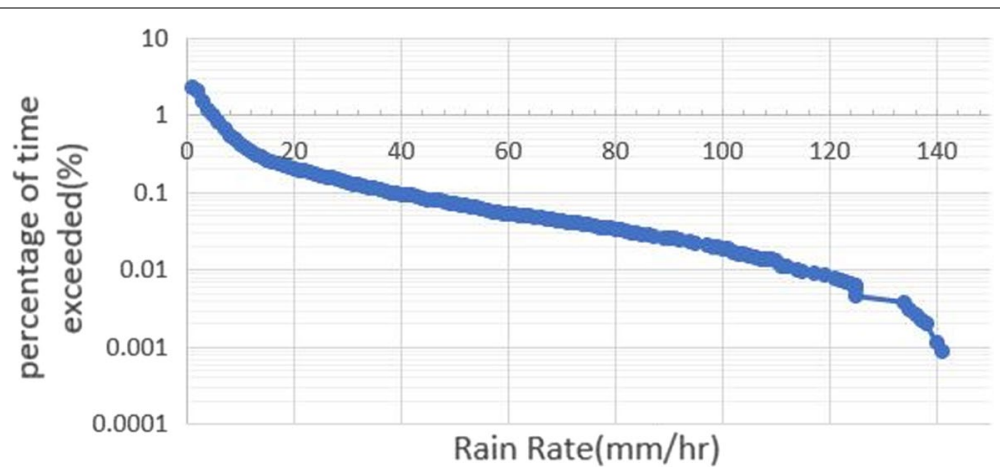

Fig. 7 Rain rate versus percentage of time exceeded for $0.01 \%\left(R_{0.01}\right)$. A diagram that which shows the relationship between rain rate and percentage of time exceeded for $0.01 \%$. Based on computation carried out using ITU-R rain-induced attenuation model, the $R_{0.01}$ is $113 \mathrm{~mm} / \mathrm{h}$ for a frequency of operation $11-\mathrm{GHz}$ and a path distance of $13.4 \mathrm{~km}$ (the distance between Jimma and Mujja)

very-low, medium, high, and very-high membership functions. The output of the neuro-fuzzy model has only one membership function, i.e. data rate.

\section{Results and discussion}

This research work has been done by employing MATLAB 2018a simulation software. In this simulation work,

- For a BER versus SNR performance simulation analysis of different modulation schemes and coding rates, 10 million random bits are exploited.

- Perfect knowledge of the channel state information (CSI) at the receiver is assumed.

- At any point of distance, the power of the signal is assumed to be more than that of the noise signal, i.e. the SNR is assumed greater than $0 \mathrm{~dB}$.

- The designed system under investigation is a single frequency carrier communication systems. 


\subsection{Rain attenuation results}

In this section, results of the collected rain data analysis are presented. $R(\mathrm{~mm} / \mathrm{h}), \gamma_{R}$, and total rain-induced attenuation at $R_{0.01}$ are analyzed for different frequency of operation above $10-\mathrm{GHz}$ and for a microwave link distance of 10-50 km (Table 5).

Figure 7 depicts rain rate versus percentage of time exceeded for $0.01 \%$. Based on computation carried out using ITU-R rain-induced attenuation model, the $R_{0.01}$ is 113 $\mathrm{mm} / \mathrm{h}$ for a frequency of operation $11-\mathrm{GHz}$ and a path distance of $13.4 \mathrm{~km}$ (the distance between Jimma and Mujja).

Figure 8 illustrates the relationship between specific attenuation $\left(\gamma_{R}\right)$ and frequency of operation at $R_{0.01}$ equals to $113 \mathrm{~mm} / \mathrm{h}$. It is seen from the graph that as the frequency of operation increase the specific attenuation also increases. This implies that frequency of operation and specific rain attenuation are directly related.

Figure 9 describes the impact of overall rain-induced attenuation at a frequency of operation above $10-\mathrm{GHz}$. The trend that is presented in the graph demonstrates that rain attenuation increases sharply as the frequency of operation above $10 \mathrm{GHz}$ tends to increase. For instance, 27.55, 45.14, 95.71, 129.19, and $145.49 \mathrm{~dB}$ rain-induced attenuation is experienced at a frequency of operation $11,15,28,38$, and $45-\mathrm{GHz}$, respectively.

In Fig. 10, the relationship between total rain-induced attenuation and radio link distance is presented. Total rain-induced attenuation rises sharply as the path between the link rise. It is observed that, 27.55, 39.47, and $76.2 \mathrm{~dB}$ attenuation are encountered at a link distance of 10,20 , and $30 \mathrm{~km}$, respectively.

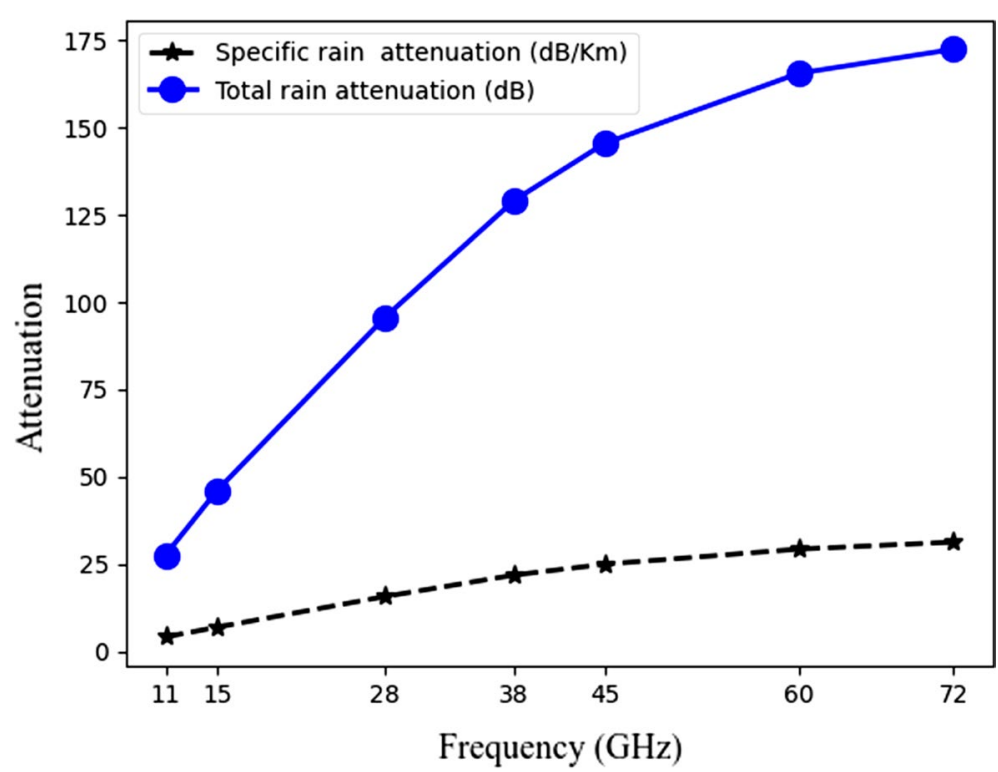

Fig. 8 Attenuation versus frequency of operation. The black line graph shows specific path attenuation $(\mathrm{dB} / \mathrm{km})$ versus frequency. This graph shows the specific path attenuation values for selected frequency of operation which are $11,15,28,38,45,60$ and $72 \mathrm{GHz}$. The blue line depicts total rain attenuation versus frequency of operation. This graph also shows the specific path attenuation values for selected frequency of operation which are $11,15,28,38,45,60$ and $72 \mathrm{GHz}$ 


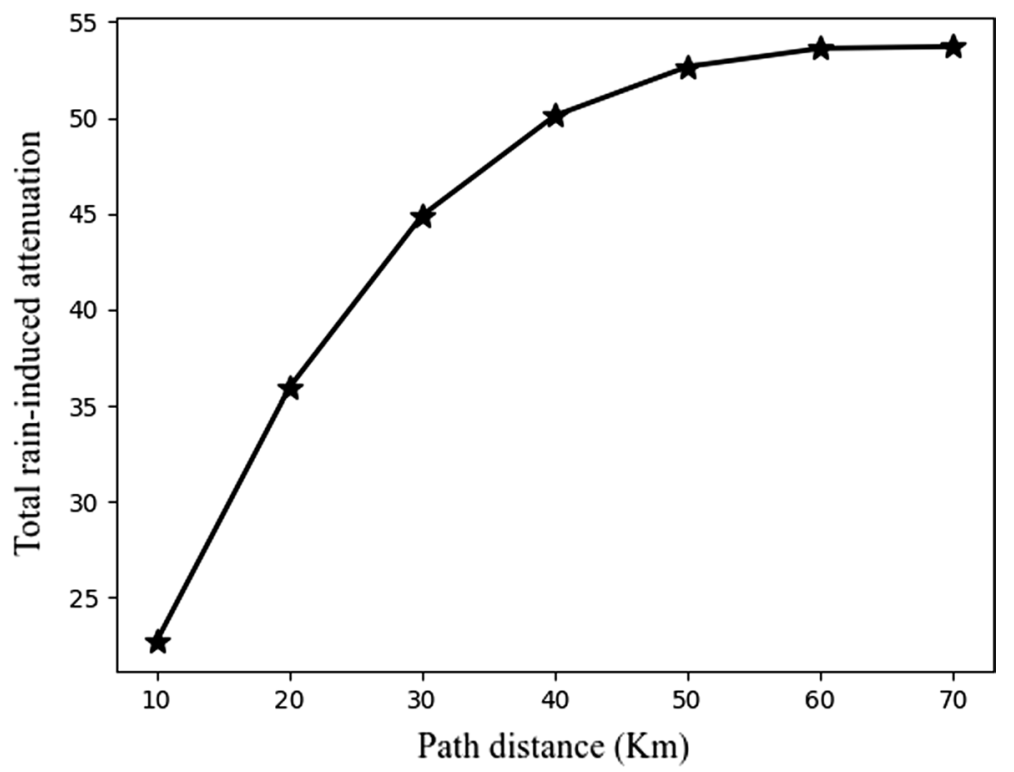

Fig. 9 Path distance versus Rain Attenuation at rain rate $R_{0.01}$ and frequency of operation $11-\mathrm{GHz}$. This graph shows the relationship between path distance and total rain induced attenuation at a rain rate of exceedance $R_{0.01}$ for a particular frequency of operation $11 \mathrm{GHz}$

Table 6 Rain attenuation related results

\begin{tabular}{lllll}
\hline Rain rate $(\mathbf{m m} / \mathbf{h})$ & $\begin{array}{l}\text { Attenuation }\left(A_{0.01}\right) \\
(\mathbf{d B})\end{array}$ & $\begin{array}{l}\text { Received signal }\left(P_{r}\right) \\
(\mathbf{d B})\end{array}$ & Fade margin $(\mathbf{d B})$ & $\mathbf{S} / \mathbf{N}(\mathbf{d B})$ \\
\hline 1 & 0.5763 & -68.7663 & 37.4337 & 31.8637 \\
5 & 1.6966 & -69.8866 & 36.3134 & 30.7434 \\
10 & 3.014 & -71.2043 & 34.9956 & 29.4256 \\
20 & 5.5354 & -73.7254 & 32.4746 & 26.9046 \\
30 & 7.9886 & -76.1786 & 30.0213 & 24.4513 \\
40 & 10.4064 & -78.5964 & 27.6035 & 22.0336 \\
50 & 12.8019 & -80.9919 & 25.2081 & 19.6381 \\
60 & 15.1818 & -83.3718 & 22.8282 & 17.2582 \\
70 & 17.5501 & -85.7401 & 20.4599 & 14.8899 \\
80 & 19.9096 & -88.0996 & 18.1004 & 12.5304 \\
90 & 22.2621 & -90.4521 & 15.7479 & 10.1779 \\
100 & 24.6088 & -92.7988 & 13.4012 & 7.8312 \\
110 & 26.9508 & -95.1408 & 11.0592 & 5.4892 \\
$\mathbf{1 1 3}$ & $\mathbf{2 7 . 6 5 2 6}$ & -95.7526 & $\mathbf{1 0 . 4 4 7 4}$ & $\mathbf{4 . 8 7 7 4}$ \\
120 & 29.2888 & -97.4788 & 8.7212 & 3.1512 \\
130 & 31.6234 & -99.8134 & 6.3866 & 0.8166 \\
\hline
\end{tabular}

Bold values indicate the numbers obtained at the rain rate of exceedance for that particular area. Rain rate $113 \mathrm{~mm} / \mathrm{hr}$ is the rain rate of exceedance $\left(R_{0.01}\right)$

\subsection{Signal level analysis}

From the computation procedure outlined in SNR calculation section, for the clear sky situation the received signal level at the receiver end using a transmitting power of 30 $\mathrm{dBm}$ is $-38.1 \mathrm{dBm}$ for the microwave link between Jimma and Muja. 


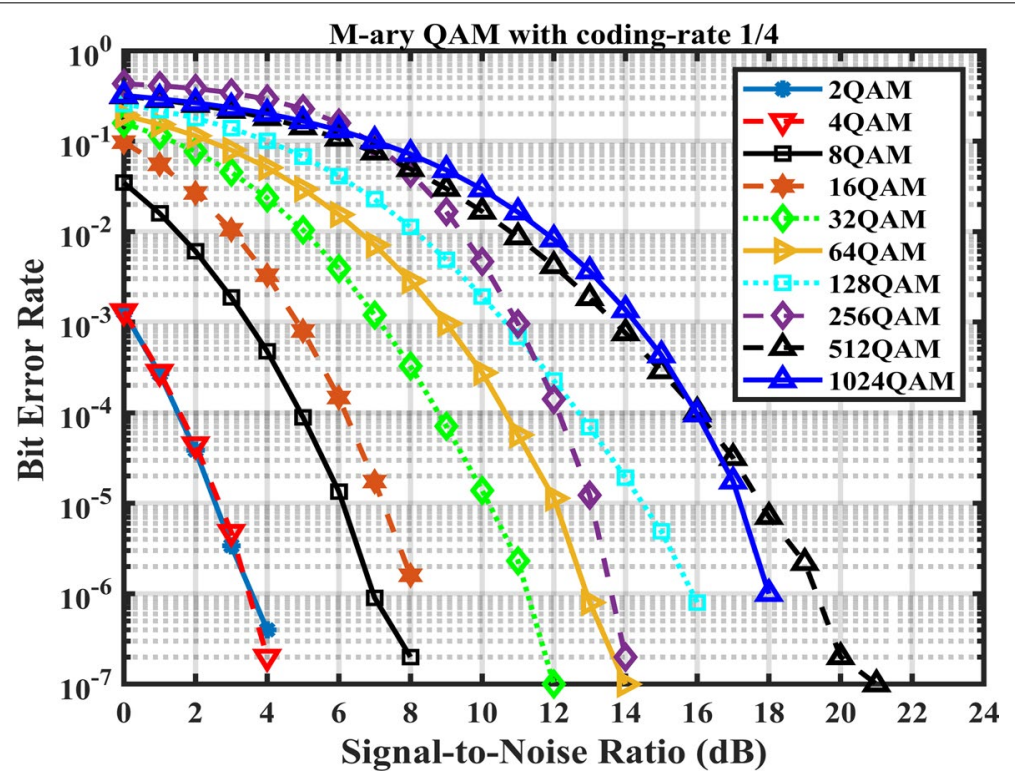

Fig. 10 BER versus SNR for different M-ary QAM with 1/4 code rate. This graph depicts the performance of different M-ary QAM with 1/4 code rate combination. The performance is described in terms of BER versus SNR

From the link profile given in Table 2, we observe that the receiver threshold signal level magnitude is $-76.2 \mathrm{dBm}$. Therefore, by applying (16), the fade margin is equal to $38.1 \mathrm{~dB}$. This implies that, if the fade depth is between 0 and $38.1 \mathrm{~dB}$, there is a faded reception due to the poor signal level. Whereas for the fade depth greater than $68.1 \mathrm{dBm}$ or $38.1 \mathrm{~dB}$, there will be network outage.

Table 6 shows that as the rain rate increases the total rain attenuation $A_{0.01}$ is increased, and the received signal level and SNR is decreasing. For instance, for rain rate 5, 25, 50, 100 , and $130 \mathrm{~mm} / \mathrm{h}$ the receiver detects a SNR value of 30.74, 25.67, 19.64, 7.83, and $0.8165 \mathrm{~dB}$, respectively. When the rain rate is above $130 \mathrm{~mm} / \mathrm{h}$, the resulting SNR at the receiving end reduced below $0 \mathrm{~dB}$. Total network outage will happen when the rain rate is above $130 \mathrm{~mm} / \mathrm{h}$ as SNR is $0 \mathrm{~dB}$. This is because the transmitted signal is too much attenuated by rain and become indistinguishable from the noise signal associated with it.

\subsection{Simulation result of ACM performance}

\subsubsection{BER performance results}

In this section, performance plots of BER versus SNR for different modulation schemes are studied with various code rates under additive white Gaussian noise (AWGN) transmission medium. Each plot in these graphs represents the BER performance of a specific modulation-code pair.

As has been seen from the graphs in Fig. 11 BER decline dramatically as the SNR tends to increase. The lower modulation order and coding rates provide better performance with the wireless condition having high rain rate (i.e, low SNR). On the contrary, when the received SNR is high at a clear sky condition, a higher-order modulation and higher coding rate schemes give a good performance. 


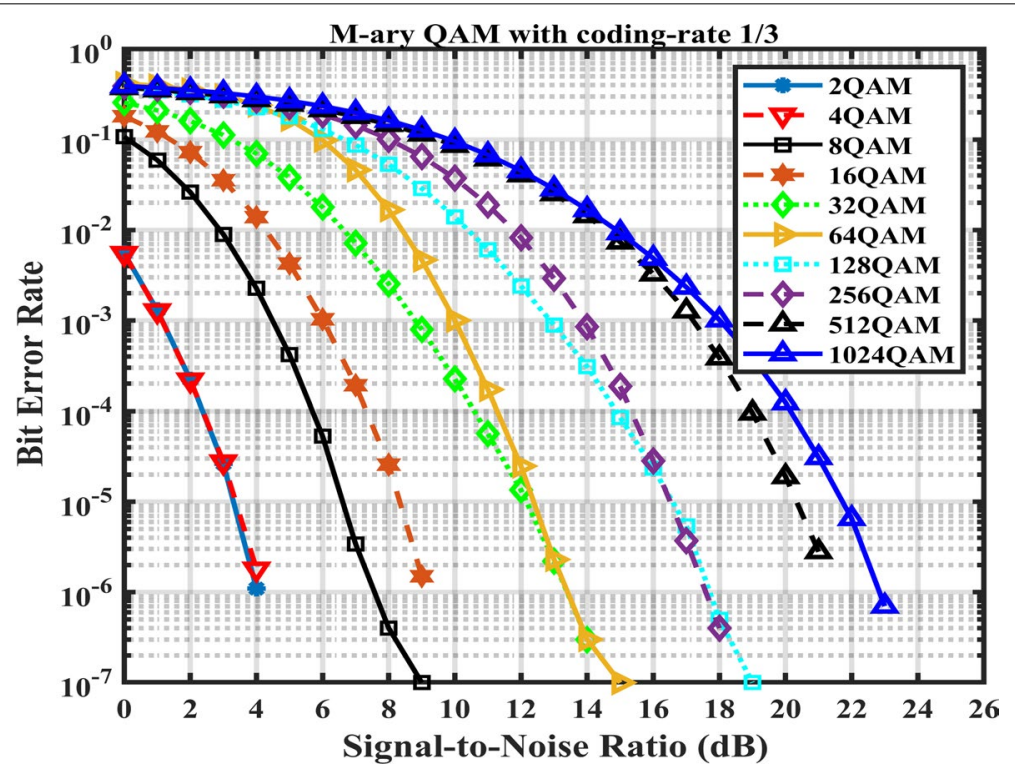

Fig. 11 BER versus SNR for different M-ary QAM with $1 / 3$ code rate. This graph depicts the performance of different M-ary QAM with 1/3 code rate combination. The performance is described in terms of BER versus SNR

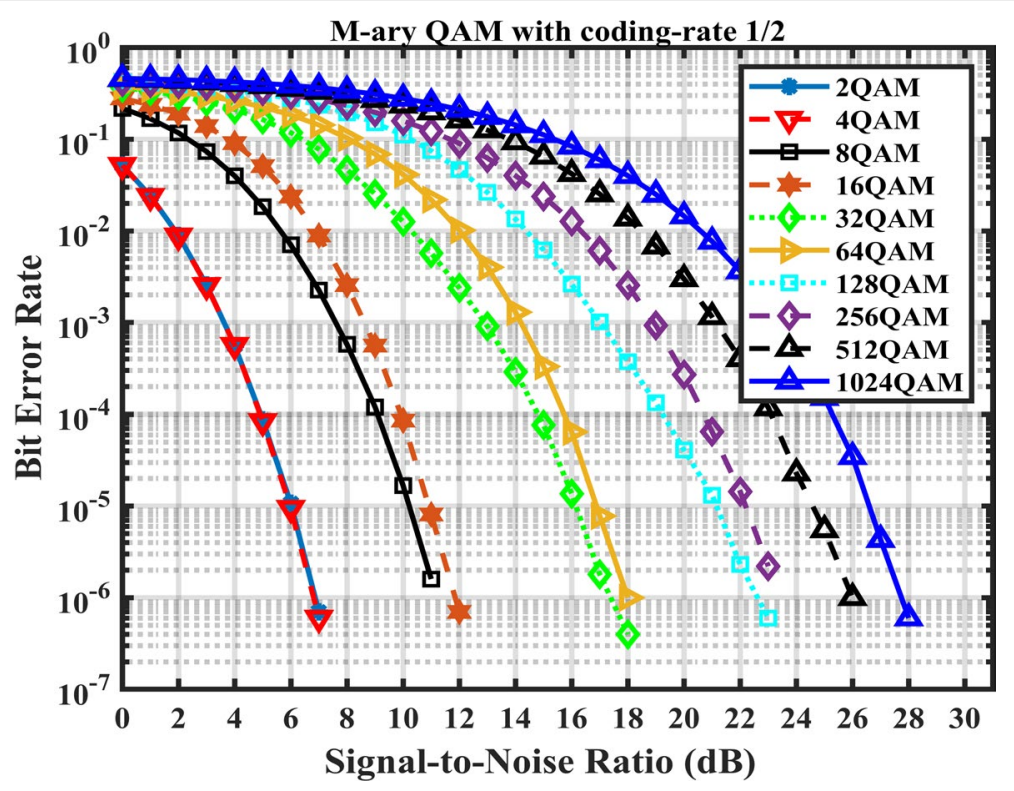

Fig. 12 BER versus SNR for different M-ary QAM with 1/2 code rate. This graph depicts the performance of different M-ary QAM with 1/2 code rate combination. The performance is described in terms of BER versus SNR

However, the plots of BER performance for modulation schemes 2 QAM and 4 QAM are overlapped. As it is clearly indicated, in ACM technique design, a modulation scheme having a better spectral efficiency over the same SNR range is selected. For this reason, 2 QAM is not used in the neuro-fuzzy-based ACM fade mitigation implementation. 
Figure 12 demonstrate SNR versus BER graphs for different M-ary QAM with 1/3 coding rate. In order to achieve a target QoS, higher SNR is required with a $1 / 3$ coding rate compared to forward error correction (FEC) of $1 / 4$ coding rate. For instance, for the 1024 QAM modulation scheme to achieve a target QoS $10^{-6}$ it requires less than $20 \mathrm{~dB}$ when its FEC is $1 / 4$ and more than $20 \mathrm{~dB}$ when the FEC is $1 / 3$.

As we can see from the result, for a target BER $10^{-5}$ and below, for the same values of SNR utilizing 64 and 256 QAM outperform 32 and 128 QAM, respectively in terms of spectral efficiency.

The BER performance for various modulation schemes with $1 / 2$ coding rate under the AWGN channel is shown in Fig. 12. The BER curves indicate that increasing the code rate increases the required SNR for a system to operate in a predetermined QoS. The BER performance comparison for various M-ary QAM using rate 2/3 and 3/4 convolutional codes are shown in Figs. 13 and 14, respectively. In general, each modulation scheme with code rate $2 / 3$ and $3 / 4$, as the SNR increases the higher modulation order performs better than the lower one for the same target BER.

The selection of the modulation order and the coding rate depends on the quality of the wireless channel. The bandwidth-efficient modulation and coding techniques are used during a good channel condition. On the other hand, a lower coding and modulation scheme are used to improve the BER performance for less SNR. For example, for SNR of $20 \mathrm{~dB}$ and target BER of $10^{-4}, 64$ QAM with $2 / 3$ and 128 QAM with a 3/4 code rate can be employed to improve capacity and maintain the link.

From the rain data analysis, it is known that for less rain conditions, in which the rain rate is between 5 to $25 \mathrm{~mm} / \mathrm{h}$, the SNR is degraded less. In this range of rain rate, the SNR is in the range $25-30 \mathrm{~dB}$. This implies that using a modulation scheme like 128, 256, and 512 QAM is appropriate to achieve a high data rate. On the contrary, for the rain

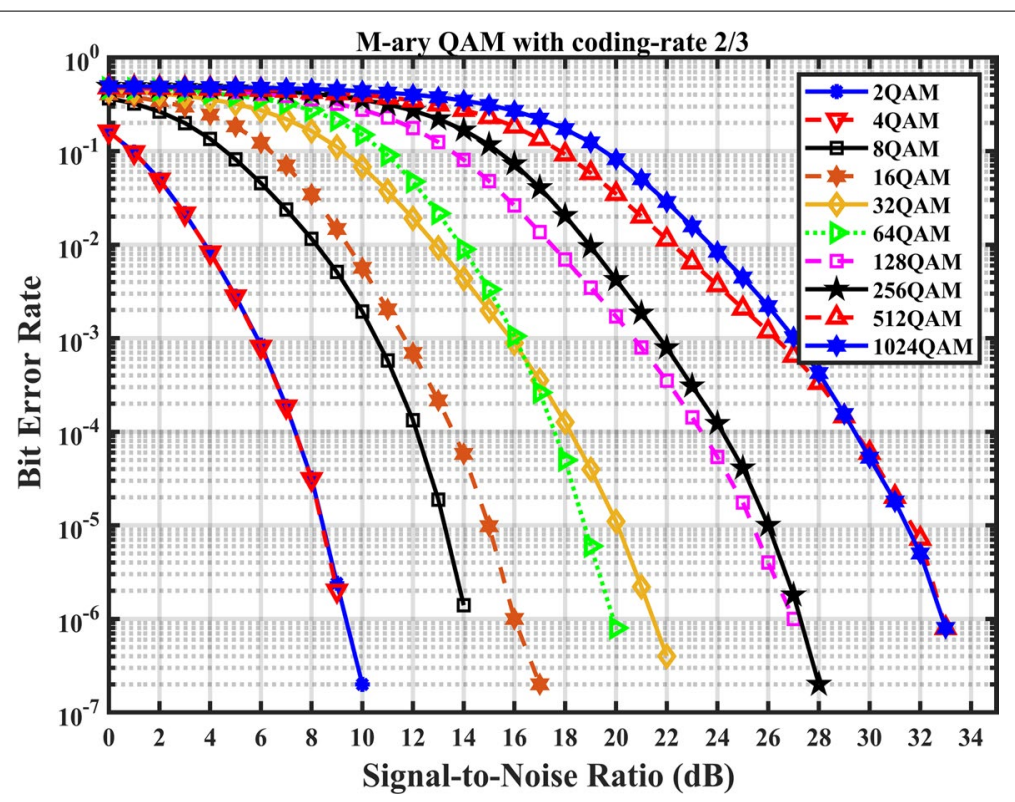

Fig. 13 BER versus SNR for different M-ary QAM with $2 / 3$ code rate. This graph depicts the performance of different M-ary QAM with 2/3 code rate combination. The performance is described in terms of BER versus SNR 


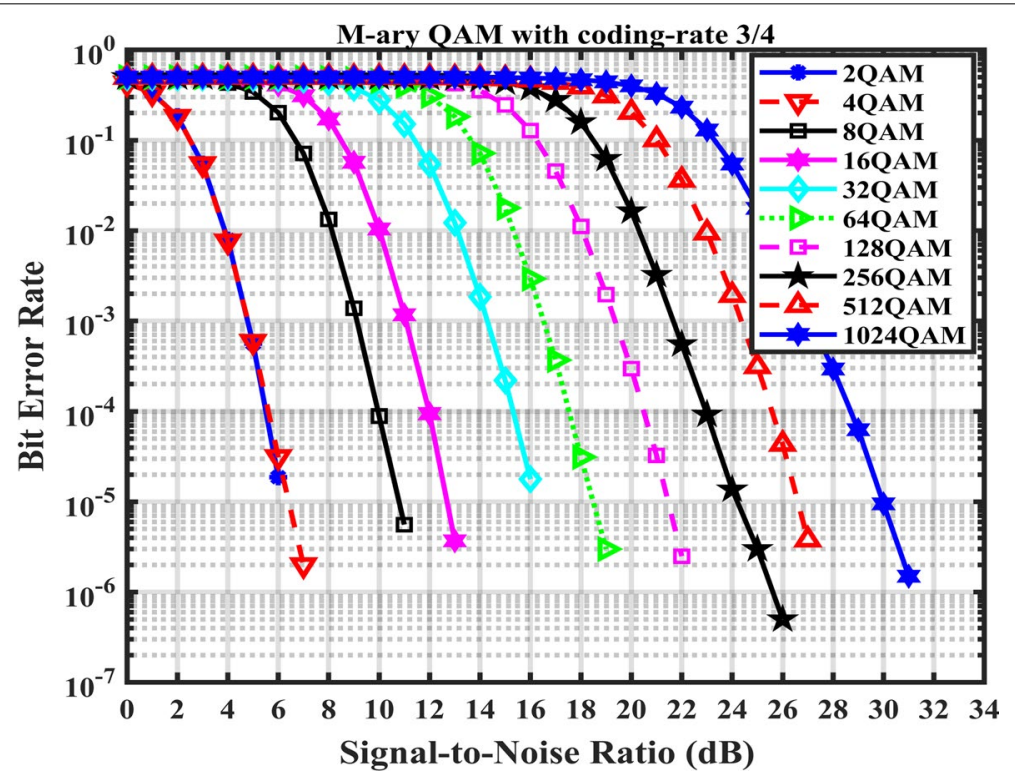

Fig. 14 BER versus SNR for different M-ary QAM with $3 / 4$ code rate. This graph depicts the performance of different M-ary QAM with 3/4 code rate combination. The performance is described in terms of BER versus SNR

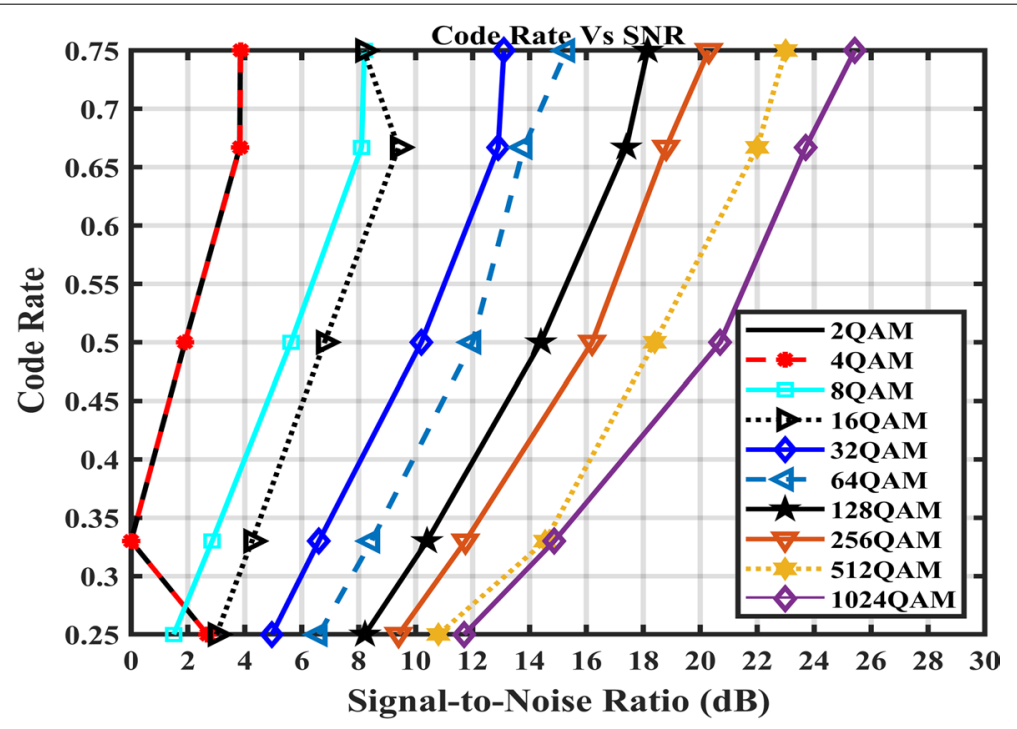

Fig. 15 Code rate versus SNR for different modulation schemes for target bit error rate of $10^{-2}$. In this graph the effect of channel coding at a desired BER of $10^{-2}$ is presented by showing the performance of different M-ary QAM scheme with respect to code rate of $1 / 4,1 / 3,1 / 2,2 / 3$, and. $3 / 4$ over a range of SNR values

rate above $105 \mathrm{~mm} / \mathrm{h}$, the SNR is reduced and become below $6 \mathrm{~dB}$ which is a deep fade condition. In this situation, it is recommended to use a lower order modulation scheme like 2, 4, and 8 QAM along with a low coding rate like $1 / 4$ and $1 / 3$. 


\subsubsection{Effect of channel coding}

The designed ACM system performance due to different FEC is analyzed below for a target BER $10^{-2}$ and $10^{-5}$. BER $10^{-5}$ is usually taken as a comparison of various modulation schemes [30] in digital communication systems. Thus, in this section comparison of different QAM modulation schemes with respect to coding rate and SNR is presented.

Figure 15 shows the required SNR to meet the target $B E R=10^{-2}$ for various constellation sizes with $1 / 4,1 / 3,1 / 2,2 / 3$, and $3 / 4$ code rates. The higher modulation schemes require higher SNR. In addition, increasing the code rate increases the required SNR to meet the target QoS for each modulation order.

Lower order modulation schemes (4, 8, and 16 QAM) can perform at lower SNR values with the indicated coding rates. However, higher-order modulation such as, 512 and 1024 QAM do not operate for lower SNR values. In other words, to meet a target BER, higher modulation and coding is used during a good channel condition. Conversely, utilization of low order modulation schemes are better to maintain link availability when the channel condition becomes attenuated severely .

Figure 16 shows the plots of the required SNR to meet the target BER of $10^{-5}$ for various modulation schemes with various code rates. Since $B E R=10^{-5}$ is very much lower than $\mathrm{BER}=10^{-2}$ it is expected that the SNR required to achieve $\mathrm{BER}=10^{-5}$ is higher than that of BER $=10^{-5}$.

Figures 17 and 18 show the bit error rate comparison of 16 QAM and 256 QAM with different coding rates respectively. For the same modulation order, the BER performance varies with the coding rate. By reducing the code rate, less SNR is required to meet the desired target BER.

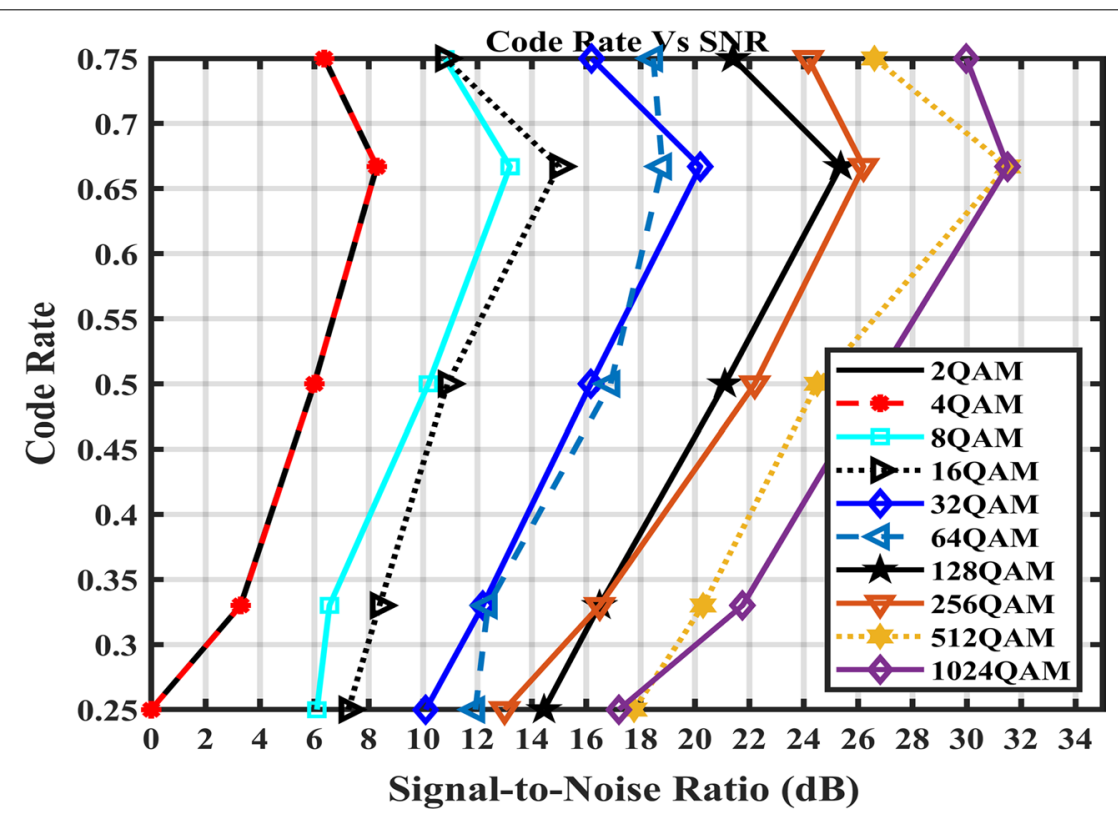

Fig. 16 Code rate versus SNR for different modulation schemes for target bit error rate of $10^{-5}$. In this graph the effect of channel coding at a desired BER of $10^{-5}$ is presented by showing the performance of different M-ary QAM scheme with respect to code rate of $1 / 4,1 / 3,1 / 2,2 / 3$, and $3 / 4$ over a range of SNR values 


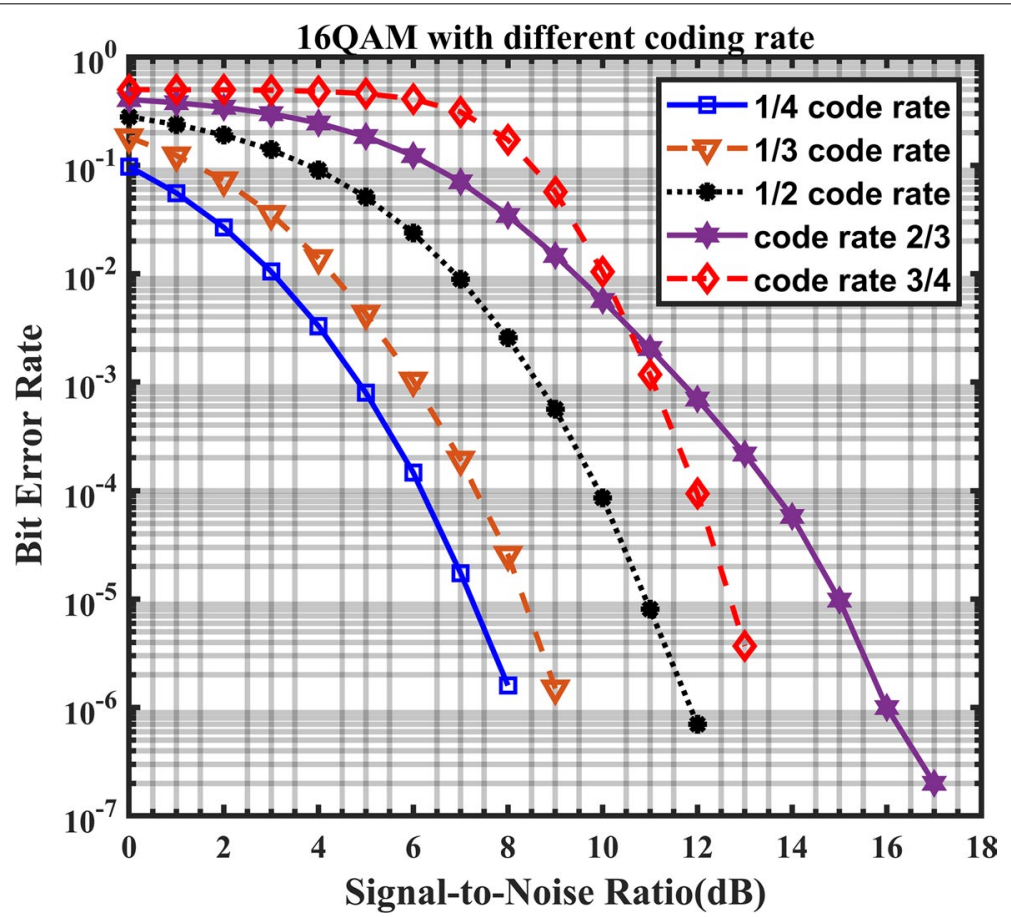

Fig. 17 BER versus SNR for 16QAM for different coding rate. In this graph the performance of a combination of16 QAM with code rate of $1 / 4,1 / 3,1 / 2,2 / 3$, and $3 / 4$ is shown. The performance is depicted as BER versus SNR

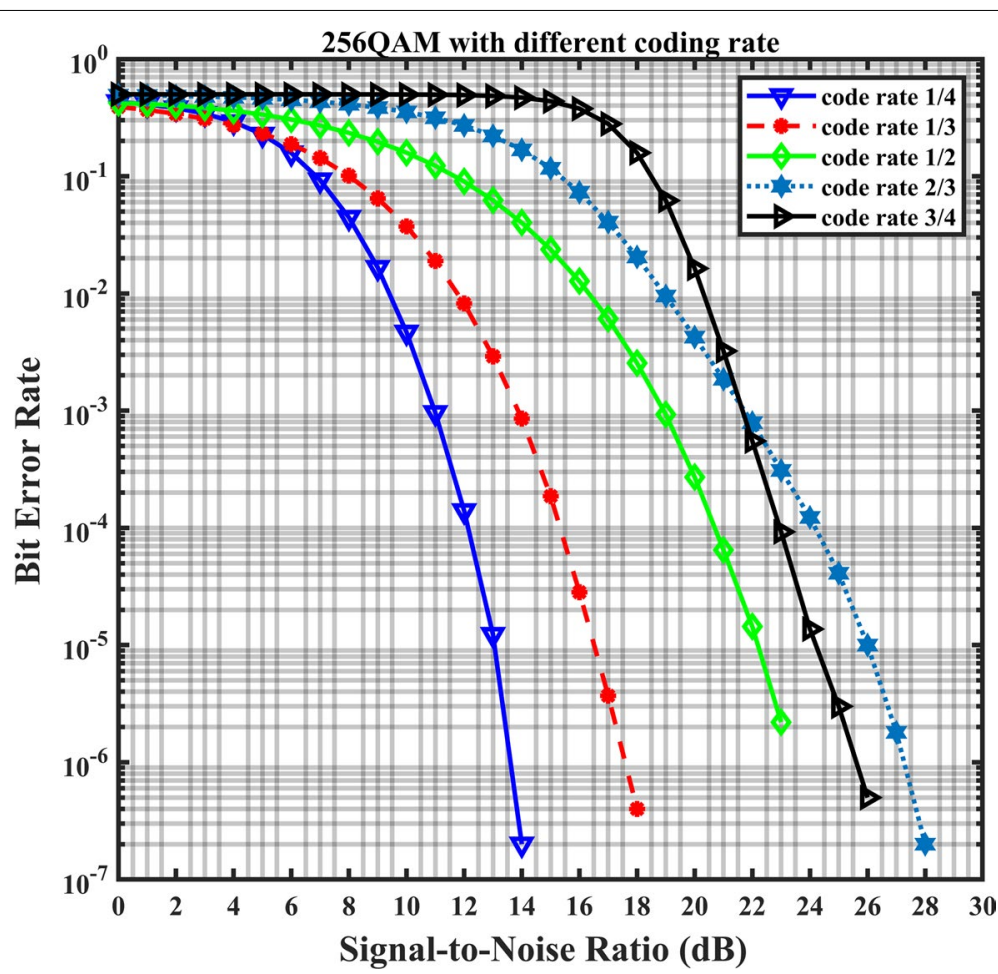

Fig. 18 BER versus SNR for 256 QAM for different coding rate. In this graph the performance of a combination of 16 QAM with code rate of $1 / 4,1 / 3,1 / 2,2 / 3$, and $3 / 4$ is shown. The performance is depicted as BER versus SNR 
For a low QoS less SNR is required compared to high QoS for the same codemodulation pair. For example, for 16 QAM with $1 / 2$ code rate, 6.8, 8.6, 9.9, 10.9, and 11.9- $\mathrm{dB}$ SNR is required to meet the bit error rate of $10^{-2}, 10^{-3}, 10^{-4}, 10^{-5}$, and $10^{-6}$, respectively as it is seen from Fig. 18. Whereas, from Fig. 19 we can see that $16.2,18.9,20.65$, and 22.2- $\mathrm{dB}$ is required to achieve a QoS $10^{-2}, 10^{-3}, 10^{-4}$, and $10^{-5}$, respectively.

As we can see in Figs. 17 and 18, for a target bit error $10^{-3}$ and less the performance of FEC $3 / 4$ is better than FEC $2 / 3$ in terms of achieving high spectral efficiency for the same value of SNR. For instance, to achieve a target BER $10^{-5}$ a SNR less than 10.8 and $25-\mathrm{dB}$ is required for 16 and 256 QAM with FEC 3/4 respectively; whereas 15 and 26.6-dB required for 16 and 256 QAM with FEC $2 / 3$.

To achieve a low BER, it is necessary to have good reception at the receiving end which results in high SNR. This can be satisfied when the channel condition is non-fading or clear sky. For the link between Jimma and Muja the calculated SNR is $32.5 \mathrm{~dB}$. In this channel quality, high quality of service and a high data rate can be achieved by employing a larger constellation size modulation scheme i.e 256, 512, and 102 QAM with a high coding rate like $2 / 3$ and $3 / 4$. Even if there is a degradation of the received signal when there is rain in between this link, it can achieve a high data rate in a rain rate below $20-\mathrm{mm} / \mathrm{h}$.

\subsubsection{Spectral efficiency results}

The spectral efficiency with various SNR ranges for different modulation and coding techniques over the AWGN channel is presented in this section. The range of SNR switching thresholds for various modulation-code pair with target BER of $10^{-2}$ and $10^{-5}$ is shown in Tables 7 and 8 respectively. These SNR values are used to select the spectrally efficient modulation-code pair for the ACM schemes.

Table 7 Sample of spectrally efficient modulation-code pair which give a target BER of $10^{-2}$ at given range of SNR value

\begin{tabular}{llll}
\hline BER $=10^{-2}$ & & & \\
\hline SNR range (dB) & Modulation & Code rate & $\begin{array}{l}\text { Data } \\
\text { rate } \\
(\mathbf{b} / \mathbf{s} / \mathbf{H z})\end{array}$ \\
\hline$<1.9$ & 4 QAM & $1 / 2$ & 1 \\
$33-3.85$ & 16 QAM & $1 / 3$ & 1.33 \\
$3.85-6.6$ & 4 QAM & $3 / 4$ & 1.5 \\
$6.8-8.45$ & 16 QAM & $1 / 2$ & 2 \\
$9.4-11.75$ & 16 QAM & $2 / 3$ & 2.66 \\
$12-12.9$ & 64 QAM & $1 / 2$ & 3 \\
$13.8-15.3$ & 64 QAM & $2 / 3$ & 4 \\
$15.3-17.4$ & 64 QAM & $3 / 4$ & 4.5 \\
$17.4-18.1$ & 128 QAM & $3 / 4$ & 5.25 \\
$18.8-20.3$ & 256 QAM & $3 / 4$ & 5.33 \\
$20.3-23$ & 256 QAM & $3 / 4$ & 6 \\
$23-25.43$ & 512 QAM & $3 / 4$ & 6.75 \\
$>25.43$ & 1024 QAM & $3 / 4$ & 7.5 \\
\hline
\end{tabular}


Table 8 Sample of spectrally efficient modulation-code pair which give a target BER of $10^{-5}$ at given range of SNR value

\begin{tabular}{llll}
\hline BER $=10^{-5}$ & & & \\
\hline SNR range (dB) & Modulation & Code rate & $\begin{array}{l}\text { Data } \\
\text { rate } \\
\mathbf{( b / s / H z )}\end{array}$ \\
\hline $3.3-6.1$ & & $1 / 3$ & 0.66 \\
$6.1-6.37$ & 4 QAM & 14 & 0.75 \\
$6.37-10.8$ & 8 QAM & $3 / 4$ & 1.5 \\
$10.8-16.2$ & 4 QAM & $3 / 4$ & 3 \\
$16.2-18.47$ & 16 QAM & $2 / 3$ & 3.75 \\
$18.47-21.4$ & 32 QAM & $1 / 2$ & 4.5 \\
$21.4-24.17$ & 64 QAM & $3 / 4$ & 5.25 \\
$24.17-26.6$ & 128 QAM & $3 / 4$ & 6 \\
$26.6-29.98$ & 256 QAM & $3 / 4$ & 6.75 \\
$>29.98$ & 512 QAM & $3 / 4$ & 7.5 \\
\hline
\end{tabular}

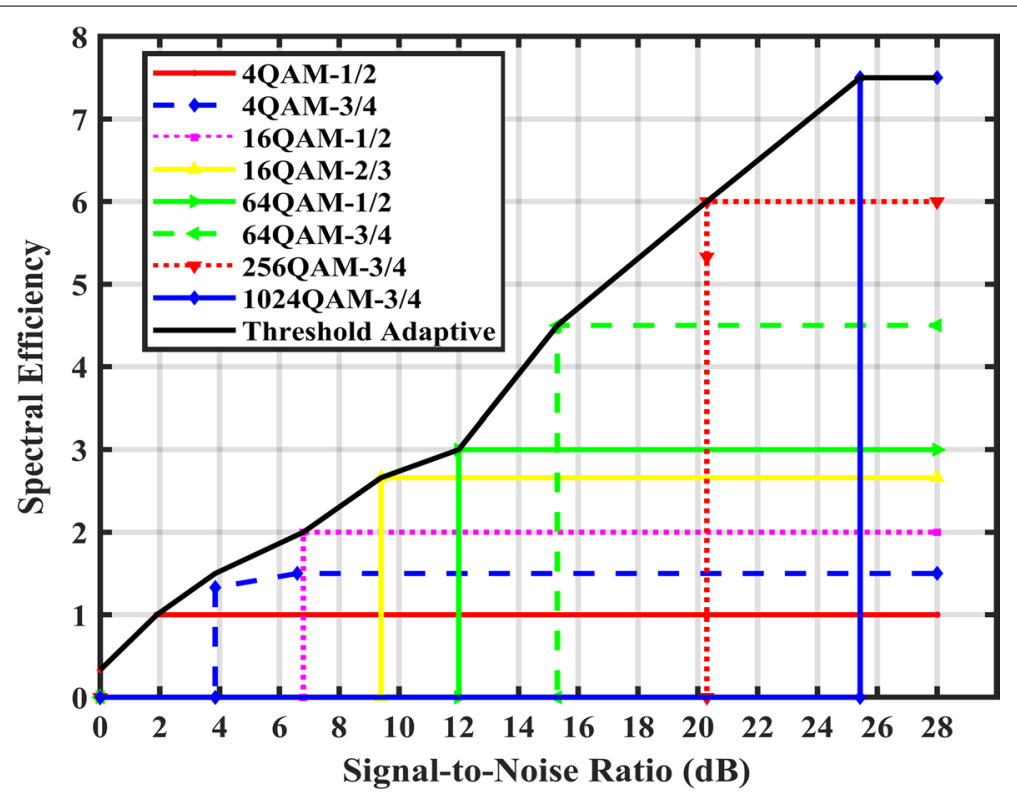

Fig. 19 Spectral efficiency versus SNR for BER of $10^{-2}$ for fixed code modulation pair and ACM schemes. The spectral efficiency $(\mathrm{b} / \mathrm{s} / \mathrm{Hz})$ performance comparison with fixed and adaptive modulation code pair techniques with a target $\mathrm{BER}$ of $10^{-2}$ is shown in this figure

The spectral efficiency $(\mathrm{b} / \mathrm{s} / \mathrm{Hz})$ performance comparison with fixed and adaptive techniques with a target BER of $10^{-2}$ is shown in Fig. 19 based on Table 7. Similarly, a spectral efficiency comparison of fixed and adaptive technique while meeting the desired BER of $10^{-5}$ is presented in Fig. 20 as tabulated in Table 8. The spectral efficiency when SNR with $3 / 4$ coding rate for $4,16,64,265$, and 1024 QAM is more than 10.8, 16.2, 21.4, 26.6 , and $29.98-\mathrm{dB}$, respectively to maintain a BER of $10^{-5}$.

What we have seen from Figs. 19 and 20 is that the spectral efficiency is proportional to the estimated SNR. In fixed coding and modulation technique, the throughput achieved at a given SNR value will not be changed even if the SNR at the receiving 


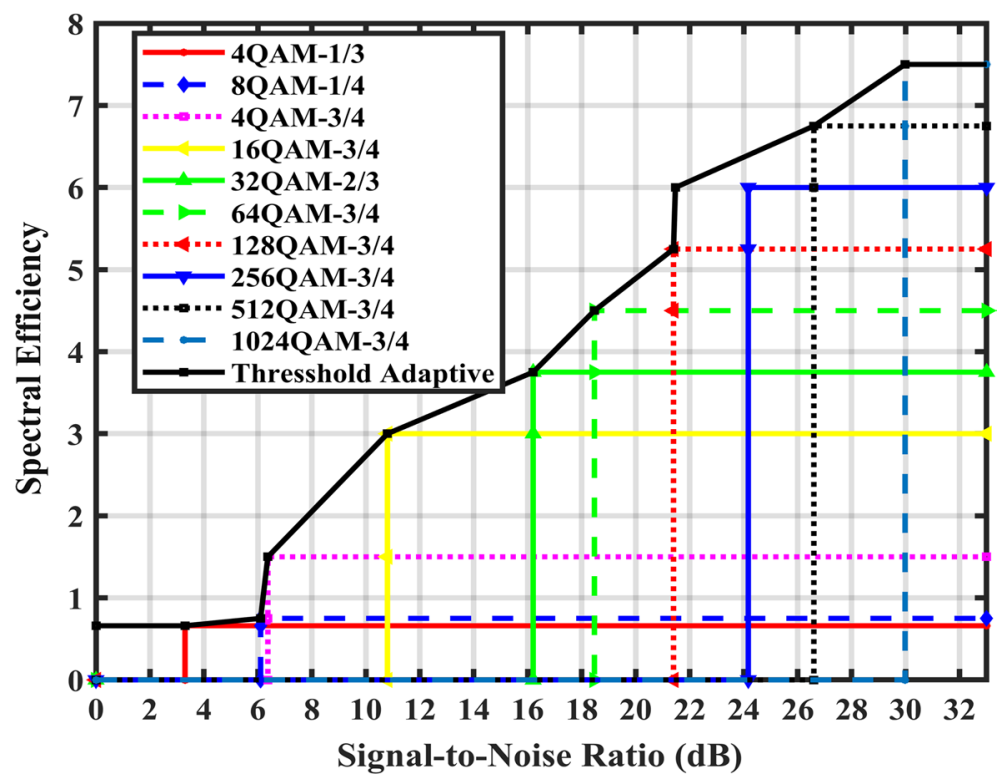

Fig. 20 Spectral efficiency versus SNR for BER of $10^{-5}$ for fixed code modulation pair and ACM schemes. The spectral efficiency $(\mathrm{b} / \mathrm{s} / \mathrm{Hz})$ performance comparison with fixed and adaptive modulation code pair techniques with a target $B E R$ of $10^{-5}$ is shown in this figure

end tends to vary. Hence, in fixed coding and modulation system there is no chance to exploit the good SNR value received at the receiver due to good channel condition. However, increasing the constellation size (modulation order) with coding rate increase the performance of wireless systems in ACM technique. For example, 256 QAM with 3/4 coding rate has a higher throughput than the lower code-modulation pair schemes such as 4 QAM-3/4. This tells us that when the channel condition is good, the receiver receives higher SNR. In this case, it is better to use a larger constellation size of a modulation scheme with a higher coding rate. In contrast, while the channel quality is bad, the receiver receive low SNR level. Here, using more robust modulation scheme having small constellation size with low coding rate is mandatory to sustain the link.

In other word, 512 QAM and 1024 QAM with coding rate $2 / 3$ and $3 / 4$ is used when the rain rate is less (i.e $0-25 \mathrm{~mm} / \mathrm{h}$ ) to achieve a high spectral efficiency and high QoS. By using a medium constellation size modulation order 64,128 , and 256 QAM, with coding rate $1 / 2$ and $2 / 3$, the link availability is sustained and achieve a moderate spectral efficiency in the channel condition when the rain rate is between $30-80 \mathrm{~mm} / \mathrm{h}$. However, while the rain rate is increased above $80 \mathrm{~mm} / \mathrm{h}$ the adaptive coding and modulation system is forced to use a lower order modulation scheme (i.e 32, 16, and 8 QAM) to keep the wireless link alive by reducing the data rate. Finally, when the channel condition becomes worse, more priority is given to sustaining the link availability than achieving high spectral efficiency. Thus, for a rain rate above $110 \mathrm{~mm} / \mathrm{h}$, a more robust lower constellation size modulation type with a coding rate $1 / 4$ is used. 
Table 9 Neuro-fuzzy parameters and their corresponding values

\begin{tabular}{lll}
\hline & ACM parameters & Values \\
\hline Input variables & SNR & $0-35 \mathrm{~dB}$ \\
& Modulation & 4 QAM, 8 QAM, 16 QAM, \\
& Scheme & $32 \mathrm{QAM}, 64 \mathrm{QAM}, 128 \mathrm{QAM}$, \\
& $256 \mathrm{QAM}, 512 \mathrm{QAM}, 1024 \mathrm{QA}$ \\
& Coding & $1 / 4,1 / 3,1 / 2$, \\
Output variables & Rate & $2 / 3$, and 3/4 \\
& Spectral & $0.5-7.5$ \\
& Efficiency & $\mathrm{b} / \mathrm{s} / \mathrm{Hz}$ \\
\hline
\end{tabular}

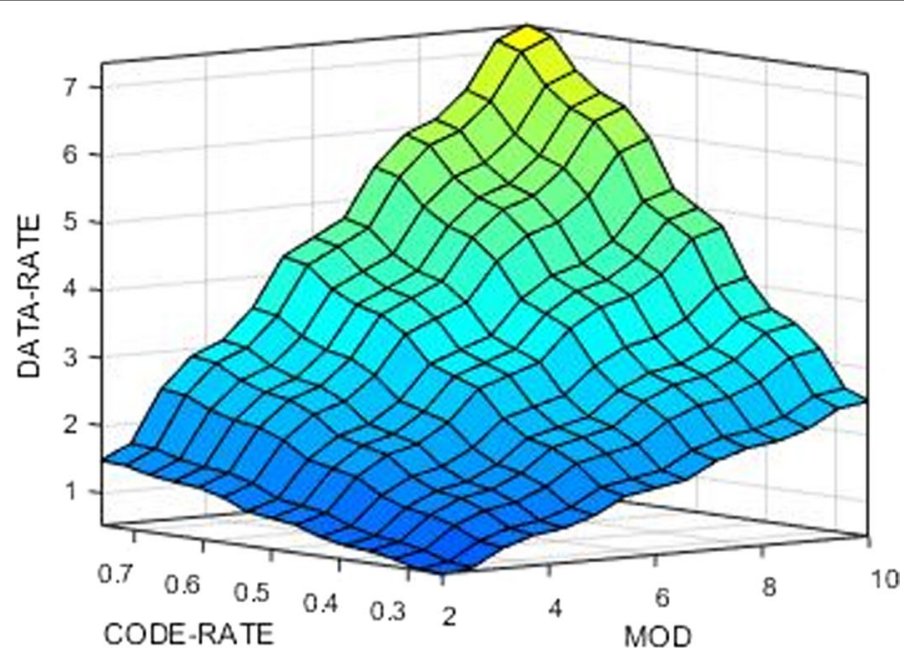

Fig. 21 ANFIS surface view for MOD versus Code-Rate. This figure shows the 3D ANFIS surface views of the Modulation order and coding rate combination result. The upper yellow surface indicates a high order modulation schemes with high coding rate $(2 / 3$ and $3 / 4)$ to give a higher data rate.The surface with blue color indicates a low data rate resulted from a low order modulation schemes and a low coding rate

\subsection{Parameter selection to maximize spectral efficiency}

In this paper, ACM of a single frequency carrier system is simulated under an AWGN channel. For each given SNR a BER is computed. The given SNR is investigated for each modulation order and coding rate. Hence, the spectral efficiency of an ACM scheme for the designed single frequency carrier systems is dependent on the BER, SNR, coding rate, and modulation order.

The parameters that are used as input and output in ANFIS training along with their respective values are tabulated in Table 9.

$$
\eta=f\left(B E R, S N R, m, R_{C}\right)
$$

where $m=\log _{2}(M), \mathrm{M}$ is the modulation/constellation size and $R_{C}$ is the FEC convolutional coding rate.

It is known that from previous sections, SNR is directly dependent on the channel condition. The channel condition in turn is dependent on the rain rate that is found in 


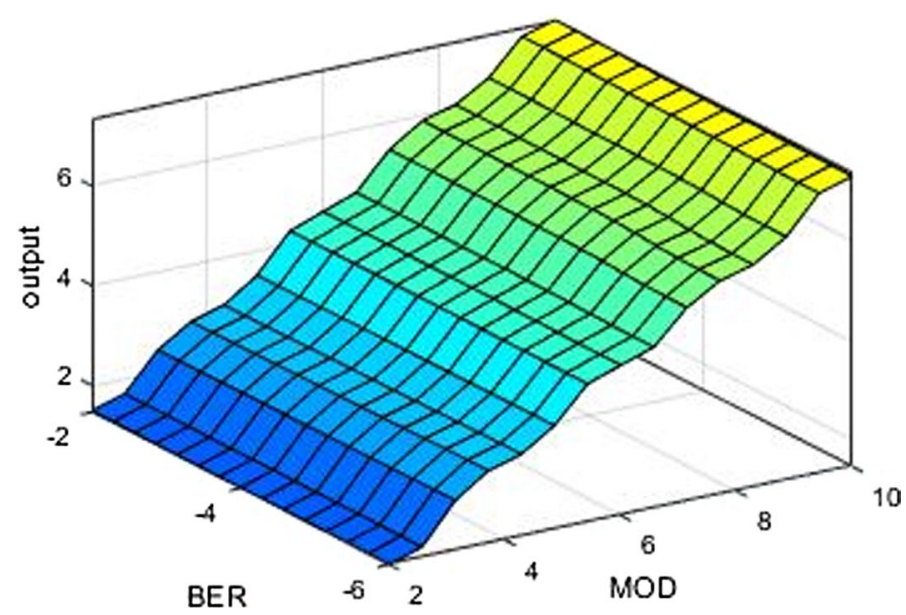

Fig. 22 ANFIS surface view for BER versus MOD. This figure shows the 3D ANFIS surface views of the QAM modulation order and BER combination result. The upper yellow surface indicates a high order modulation schemes in achieving the indicated values of BER. The surface with blue color indicates a low data rate resulted from a low order modulation schemes for various target BER

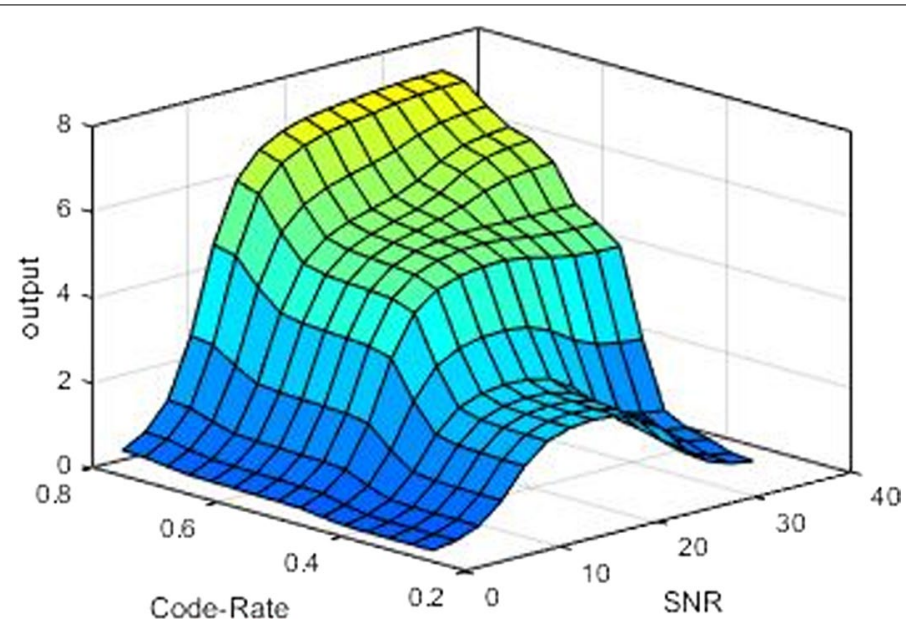

Fig. 23 ANFIS surface view for SNR versus Code-Rate. This figure shows the 3D ANFIS surface views of the signal-to-noise ratio and coding rate combination result. The upper yellow surface indicates a better data rate resulted from a high SNR value at higher coding rate. The surface with blue color indicates a low data rate resulted from a low order modulation schemes for various target BER

the path between the transmitter and receiver of the link. As a result, rain rate is an indirect input parameter to the neuro-fuzzy-based ACM system.

\subsection{Neuro-fuzzy-based system results}

The value of epoch and error tolerance is set to 50 and $10^{-4}$ as tolerance error and in the ANFIS training process. The output is selected based on the constructed 225 fuzzy rules. In this system, the if-then rules are used to make the decision in data rate optimization. The ANFIS rule viewer gives a better description of all fuzzy rules. Figures 21, 22, 23 and 24 show different surface views. These 3D curves represent 


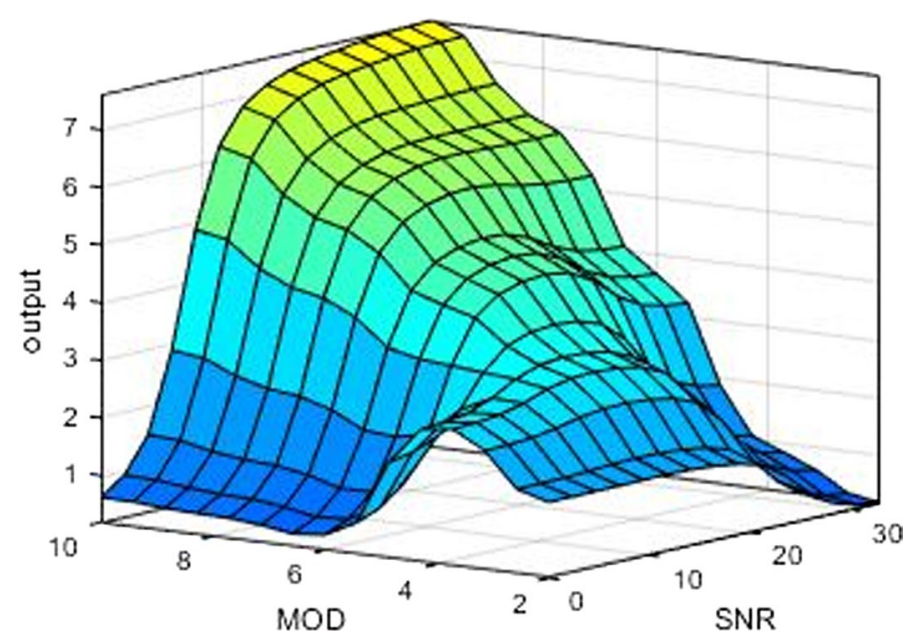

Fig. 24 ANFIS surface view for SNR versus Modulation. This figure shows the 3D ANFIS surface views of the signal-to-noise ratio and different QAM modulation schemes combination result. The upper yellow surface indicates a better data rate resulted from a high SNR value at higher order QAM modulation schemes. The surface with blue color indicates a low data rate resulted from a low order QAM modulation schemes for a range of SNR values

the mapping of input variables against output variable. In other words, it dictates the smoothness and correlation between the input variables to select the desired output at a particular time depending on the quality of the channel. The output axis describes the spectral efficiency result of the two other input combinations. The surface colors indicate the level of the output. As shown in all figures, the yellow, light blue, and dark blue colors show the data rate is high, average, and low, respectively.

Figure 21 illustrates the modulation order-coding rate combination to attain the best achievable data rate for a given SNR and BER value. It is seen that as both the modulation order and coding rate increases simultaneously, the output data rate increases too.

The two controlled input variables SNR and coding rate are fixed to a value of $16.5 \mathrm{~dB}$ and 0.75 , respectively while evaluating BER versus modulation relationship in Fig. 22.

The step-size like graph in Fig. 23 implies that as the coding rate increases the achievable data rate also increases simultaneously. The lower coding rate, i.e. 1/2 and $1 / 3$ allows to achieve a lower data rate as we can see from blue color.Where as a higher data rate can be achieved using high coding rate (i.e. $2 / 3$ and $3 / 4$ ) as it is seen from yellow color.

In Fig. 24, the SNR and modulation ANFIS input relationship, the blue color tells that for high SNR value (clear sky channel condition) lower order modulation scheme is not selected. In this type of channel condition, higher order modulation scheme like 256, 512, and 1024 QAM are employed to attain higher data rate as it is indicated by yellow color region. On the contrary, when the channel quality is lower (rainy time) a lower constellation size modulation scheme (4, 8, and 16 QAM) are used to sustain link availability by penalizing data rate. 
Table 10 Data rate comparison of the proposed scheme to different existing models at atarget BER $=10^{-2}$

\begin{tabular}{lllllll}
\hline Scheme & \multicolumn{2}{l}{$\mathbf{S N R}$} \\
\cline { 2 - 7 } & $\mathbf{5 ~ d B}$ & $\mathbf{1 0 ~ d B}$ & $\mathbf{1 5} \mathbf{~ d B}$ & $\mathbf{2 0 ~} \mathbf{~ B}$ & $\mathbf{2 5} \mathbf{~ d B}$ & $\mathbf{3 5} \mathbf{~ d B}$ \\
\hline Neuro-Fuzzy based ACM & 1.6 & 5.84 & 7.2 & 7.5 & 7.5 & 7.5 \\
Fuzzy based ACM & 1.4 & 3.9 & 5.8 & 6.9 & 7.2 & 7.5 \\
Switching threshold based ACM & 1.5 & 2.66 & 5.25 & 6 & 6.75 & 7.5 \\
\hline
\end{tabular}

\subsection{Performance comparison of the ANFIS to various schemes}

Simulation results illustrate that the proposed system performs well compared to other techniques in terms of spectral efficiency or data rate for a target BER of $10^{-2}$ and constant transmit power. Thus, system performance is maximized by changing the code rate-modulation scheme pair such that the BER and total transmitted power kept below certain thresholds.

Table 10 shows the data rate comparison of the proposed scheme to different existing models for SNR $5,10,15,20,25$, and $35-\mathrm{dB}$. At SNR range 5 to $35 \mathrm{~dB}$, a neurofuzzy-based ACM shows superiority in spectral efficiency compared to both fuzzy logic-based ACM and switching threshold-based ACM. This implies that the adaptation capability of neuro-fuzzy-based ACM system in response to varying channel condition is supreme.

\section{Conclusion}

In this study, a two-year rain intensity data recorded at a minute sampling rate,which was obtained from a measuring device installed at Jimma Institute of Technology, Jimma University, Ethiopia, was utilized. And the $R_{0.01}$ was then calculated using this locally recorded rain data. Besides, the specific rain and total path rain attenuation was estimated for a particular microwave link (between Jimma and Mujja, Ethiopia) by employing ITU-R models. Following this, the mitigation of this rain attenuation was determined via the neuro-fuzzy-based ACM system. The performance of neuro-fuzzy-based ACM systems in terms of spectral efficiency and BER using various coding rates and QAM modulation schemes under the AWGN channel was analyzed and compared to fixed and adaptive techniques.

From the present result, the SNR at the receiving end of the particular link (between Jimma and Mujja) of Ethiopia was found to be $32.5-\mathrm{dB}$ in the clear sky (non-rain time) condition. On the other hand, for the rain rate above $130 \mathrm{~mm} / \mathrm{h}$, there will be a total link outage. The BER performance is improved by using the FEC coding rate. However, selecting a lower code rate can reduce spectral efficiency. During good quality of channel, higher coding and modulation orders such as 256, 512, and 1024 QAM with coding rate $2 / 3$ and $3 / 4$, can be used to improve data rate. Whereas, in rainy time (bad channel condition), the more robust modulation schemes like 4, 8, and 16 QAM (lower modulation order QAM) are better in sustaining link availability. For instance, in the rainy condition, it is better to use a lower order modulation with a lower coding rate. At a rain rate above $105 \mathrm{~mm} / \mathrm{h}$, the signal to noise ratio level is reduced to around $6 \mathrm{~dB}$. This implies that using high order modulation scheme, which demands high SNR, such as 128, 256, 
512, and 1024 QAM along with high code rate like 2/3 and 3/4 result in link reliability reduction. In the present investigation, from the MATLAB simulation output, ACM showed better system performance (in terms of spectral efficiency and achieving a target BER) compared to non-adaptive techniques. Furthermore, the adaptive performance of ANFIS-based ACM system was found more efficient than the fuzzy based and threshold ACM. The utilization of adaptive neuro-fuzzy inference system (ANFIS) as a decisionmaking mechanism gives a better result in an adaptation of the wireless channel condition compared to the traditional threshold ACM system.

\begin{abstract}
Abbreviations
ACM: Adaptive coding and modulation; AGC: Automatic gain control; ANFIS: Adaptive network fuzzy inference system; AWGN: Additive white Gaussian noise; BER: Bit error rate; CSI: Channel state information; FEC: Forward error correction; FIS: Fuzzy inference system; FMT: Fade mitigation technique; FRBS: Fuzzy rule-base system; GHz: Giga Hertz; GRBF-NN: Gaussian radial bases function-neural network; I/O: Input/output; ITU: International Telecommunication Union; ITU-R: International Telecommunication Union-Recommendations; LOS: Line-of-sight; MCP: Modulation code pair; MF: Membership function; ModCod: Modulation and coding; MPAM: M-ary pulse amplitude modulation; MPSK: M-ary phase shift keying; MQAM: M-ary quadrature amplitude modulation; NN: Neural network; OFDM: Orthogonal frequency division multiplexing; PAM: Phase amplitude modulation; PSK: Phase shift keying; DPSK: Differential phase shift keying; QAM: Quadrature amplitude modulation; QoS: Quality of service; QPSK: Quadrature phase shift keying; RBFNN: Rule-base fuzzy neural network; RIA: Rain induced attenuation; SNR: Signal-to noise ratio.
\end{abstract}

\title{
Authors' contributions
}

All authors contributed to the study conception and design. Data collection was done by FD. Methodology design were done by EW and FD. Original draft preparation including review and editing was done by EW. The study was supervised by FD. Both authors read and approved the final manuscript.

\section{Funding}

This work was part of the research project granted by Adama Science and Technology University (ASTU).

\section{Availability of data and materials}

The data set used and/or analysed during the current study are available from the corresponding author on reasonable request.

\section{Declarations}

Ethics approval and consent to participate

Not applicable.

\section{Competing interests}

The authors declare that there is no competing interests.

\section{Author details}

${ }^{1}$ School of Electrical and Computer Engineering, Haramaya Institute of Technology (HiT), Haramaya University, 138 Haramaya, Oromiya, Ethiopia. ${ }^{2}$ Department of Electronics and Communication Engineering, School of Electrical Engineering and Computing, Adama Science and Technology University, 1888 Adama, Oromiya, Ethiopia.

Received: 28 October 2021 Accepted: 27 December 2021

Published online: 20 January 2022

\section{References}

1. R.L. Freeman, Radio System Design for Telecommunications, 3rd edn. (Wiley, Hoboken, 2006)

2. H. Sizun, Radio Wave Propagation for Telecommunication Applications (Springer, Paris, 2003)

3. R.K. Crane, Propagation Handbook for Wireless Communication System Design (CRC Press, Boca Raton, 2003)

4. F.D. Diba, Radio Wave Propagation Modeling under Precipitation and Clear-air at Microwave and Millimetric Bands over Wireless Links in the Horn of Africa, PhD, Electronic Engineering, College of Agriculture, Engineering and Science, University of KwaZulu-Natal (2017)

5. R.M. Islam, Y.A. Abdulrahman, T.A. Rahman, An improved ITU-R rain attenuation prediction model over terrestrial microwave links in tropical region. EURASIP J. Wirel. Commun. Netw. 2012(1), 189 (2012)

6. ITU-R, Propagation data and prediction methods required for the design of terrestrial lineof_-sight systems. In Recommendations. ITU-R P.530-17 (2017)

7. M. Maki, T.D. Keenanm, Y. Nakamura, K. Sasaki, Characterstics of rain drop size distribution in tropical continental squall lines observed in Darwin, Australia. J. Appl. Metrol. 40, 1393-1412 (2001) 
8. M. Ghanim, M. Alhilali, J. Din, H.Y. Lam, Rain attenuation statistics over 5 G millimetre wave links in Malaysia. In 2018 5th International Conference on Electrical Engineering, Computer Science and Informatics (EECSI), pp. 266-269 (IEEE, 2018)

9. F.D. Diba, M.A. Samad, D.-Y. Choi, The effects of rain on terrestrial links at K, Ka and E-bands in South Korea: based on supervised learning. IEEE Access 9, 9345-9355 (2021)

10. L. Luini, G. Roveda, M. Zaffaroni, M. Costa, C.G. Riva, The impact of rain on short E-band radio links for $5 G$ mobile systems: experimental results and prediction models. IEEE Trans. Antennas Propag. 68(4), 3124-3134 (2019)

11. S. Shrestha, D.-Y. Choi, Rain attenuation statistics over millimeter wave bands in South Korea. J. Atmos. Solar Terr. Phys. 152, 1-10 (2017)

12. H.Y. Lam, L. Luini, J. Din, C. Capsoni, A.D. Panagopoulos, Investigation of rain attenuation in equatorial Kuala Lumpur. IEEE Antennas Wirel. Propag. Lett. 11, 1002-1005 (2012)

13. E. Lutz, M. Werner, A. Jahn, Satellite Systems for Personal and Broadband Communications (Springer, Berlin, 2012)

14. I. T. Union. http://www.itu.Int'|

15. A.D. Panagopoulos, P.-D.M. Arapoglou, P.G. Cottis, Satellite communications at Ku, Ka, and V bands: propagation impairments and mitigation techniques. IEEE Commun. Surv. Tutor. 6(3), 2-14 (2004)

16. P. Majithiya, A. Sisodia, V. Muralidhar, V. Garg, Novel down link rain fade mitigation technique for Ka-band multibeam systems. Int. J. Satell. Commun. Netw. 25(1), 45-51 (2007)

17. ITU-R, Propagation Data and Prediction Methods Required for the Design of Earth-Space Telecommunication Systems. Rec. ITU-R P.618-13 (2017)

18. L.D.S. Mello, M. Pontes, R. De Souza, N.P. Garcia, Prediction of rain attenuation in terrestrial links using full rainfall rate distribution. Electron. Lett. 43(25), 1442-1443 (2007)

19. I. Abubakar, H.Y. Lam, J. Din, Implementation of Adaptive Coding and Modulation for satellite communication links in heavy rain regions: ad operator's perspective (2006)

20. J. Friedman, R. Sofer, Adaptive coding and modulation based on link performance prediction, ed: Google Patents (2010)

21. J. Petranovich, Mitigating the Effect of Weather on ka-Band High-Capacity Satellites (ViaSat Inc., Carlsbad, 2012)

22. S.S. Das, S. Mondal, J. Saha, K. Bandyopadhyay, Comparison of rain fade mitigation techniques using Crc and embedded pilot methods in ka-band satellite links. In 2011 IEEE 73rd Vehicular Technology Conference (VTC Spring), pp. 1-5 (IEEE, 2011)

23. Q.I. Atta-ur-Rahman, A.N. Malik, A fuzzy rule base assisted adaptive coding and modulation scheme for OFDM systems. J. Basic Appl. Sci. Res 2(5), 4843-4853 (2012)

24. K. Nagar, K. Sharma, Adaptive OFDM implementation using fuzzy inference system. IJARCSSE 3(7), 309-314 (2013)

25. K. Singh, Fuzzy logic based modified adaptive modulation implementation for performance enhancement in ofdm systems. Int. J. Intell. Syst. Appl. 8(5), 49 (2016)

26. ITU-R, Specific attenuation model for rain for use in prediction methods, Rec. ITU-R P.838-3 (2005)

27. ITU-R, Conversion of annual statistics to worst-month statistics, Recommendation ITU-R P.841-5 (2016)

28. J. Ko et al., Millimeter-wave channel measurements and analysis for statistical spatial channel model in in-building and urban environments at $28 \mathrm{GHz}$. IEEE Trans. Wirel. Commun. 16(9), 5853-5868 (2017)

29. J.S. Seybold, Introduction to RF Propagation (Wiley, Hoboken, 2005)

30. J.G. Proakis, Digital Communications, 3rd edn. (McGraw-Hill Inc., New York, 1995)

31. A. Goldsmith, Wireless Communications (Cambridge University Press, Cambridge, 2005)

32. J.-S. Jang, ANFIS: adaptive-network-based fuzzy inference system. IEEE Trans. Syst. Man Cybern. 23(3), 665-685 (1993)

33. I. Adegbindin, P. Owolawi, M. Odhiambo, Intelligent weather awareness technique for mitigating propagation impairment at SHF and EHF satellite network system in a tropical climate. SAIEE Afr. Res. J. 107(3), 136-145 (2016)

34. S.G. Temalow, Neuro-fuzzy based adaptive coding and modulation for performance improvement in OFDM wireless systems. JKUAT (2018)

\section{Publisher's Note}

Springer Nature remains neutral with regard to jurisdictional claims in published maps and institutional affiliations.

Eyob Mersha Woldamanuel was born in Harar, Ethiopia. He received the B.Sc. degree in electrical and computer engineering from Haramaya University, Ethiopia in 2014 and the M.Sc. degree in electronics and communication engineering from Adama Science and Technology University, Ethiopia in 2019. From 2015 to 2017, he was an assistant lecturer in Haramaya Institute of Technology, Haramaya University. Since 2019 he has been a lecturer and researcher with the School of Electrical and Computer Engineering, Haramaya Institute of Technology. His research interests include soft computing, massive-MIMO, radio wave propagation, microwave and satellite communication.

Feyisa Debo Diba received the B.Sc. degree in electrical engineering from Arbaminch University, Arbaminch, Ethiopia in 2006, the M.Sc. degree in Communication Engineering from Addis abab University, Addis ababa, Ethiopia in 2010, and the Ph.D. degree electronic engineering from university of KwazuluNatal, Durban, South Africa in 2017. Currently a postdoctoral fellowship at Chosun university, Gwangju, South Korea. His research interests are radio wave propagation measurements and modeling for $5 \mathrm{G}$ and beyond. 